\title{
A TWO-CLASS RETRIAL SYSTEM WITH COUPLED ORBIT QUEUES
}

\author{
IOANNIS DimitRIOU \\ Department of Mathematics, \\ University of Patras, \\ 26500 Patras, Greece \\ E-mail: idimit@math.upatras.gr
}

\begin{abstract}
We consider a single server system accepting two types of retrial customers, which arrive according to two independent Poisson streams. The service station can handle at most one customer, and in case of blocking, type $i$ customer, $i=1,2$, is routed to a separate type $i$ orbit queue of infinite capacity. Customers from the orbits try to access the server according to the constant retrial policy. We consider coupled orbit queues, and thus, when both orbit queues are non-empty, the orbit queue $i$ tries to re-dispatch a blocked customer of type $i$ to the main service station after an exponentially distributed time with rate $\mu_{i}$. If an orbit queue empties, the other orbit queue changes its re-dispatch rate from $\mu_{i}$ to $\mu_{i}^{*}$. We consider both exponential and arbitrary distributed service requirements, and show that the probability generating function of the joint stationary orbit queue length distribution can be determined using the theory of Riemann (-Hilbert) boundary value problems. For exponential service requirements, we also investigate the exact tail asymptotic behavior of the stationary joint probability distribution of the two orbits with either an idle or a busy server by using the kernel method. Performance metrics are obtained, computational issues are discussed and a simple numerical example is presented.
\end{abstract}

Keywords: asymptotic analysis, boundary value problems, coupled orbit queues, retrials

\section{INTRODUCTION}

The study of queues with coupled processors was firstly investigated by Fayolle and Iasnogorodski [29] (see also [30]), who analyzed two coupled servers in parallel with exponential service times. To gain quantitative insights about the queueing process, they derived a solution for the generating function of the stationary distribution of the underlying Markov process describing the number of jobs in both queues using the theory of Riemann-Hilbert boundary value problems.

Motivated by their work, Cohen and Boxma [17], Cohen [18-20], Boxma and Groenendijk [14], Boxma [15], Feng, Kowada, and Adachi [31], Avrachenkov, Nain, and Yechiali [7] (see also $[1,35,36,54]$, not exhaustive list) gave important generalizations and detailed analyses for various two-dimensional queueing models with the aid of the theory of Riemann (-Hilbert) boundary value problems. In particular, a detailed study of the methodological 
approach was presented in the monograph by Cohen and Boxma [17]. In [17], a coupled processor model for the case of generally distributed service times was investigated. Resing and Ormeci [50], analyzed a tandem queue with two coupled processors and determined the bivariate generating function of the stationary joint queue length distribution in terms of the solution of a Riemann-Hilbert boundary value problem. Computational issues of this problem were investigated in [55] (see also [23]). Recently, the Laplace-Stieltjes transform of the joint stationary amount of work in the system in a coupled processor model with simultaneous arrivals was studied by Badila and Resing [9].

Applications of coupled processor models arise in several situations in which coupling derives from resource sharing. Most of them can be found in telecommunications such as in data transfer in bidirectional cable and data networks [55], in bandwidth sharing of data flows [36], in the performance modeling of device-to-device communication [58], in wireless communications to model the complex interdependence among transmitters due to the shared medium and/or to interference $[10,11,13,51,56]$, as well as in assembly lines in manufacturing [2]. In cellular networks, we may consider a system consisting of two neighboring cellular base stations. A base station serves its associated users accordingly, however, if it is idle, that is, there are no active users, it may assist the neighboring base station by serving its users. Coupled processor models were also recently applied in the performance analysis of ad-hoc networks to capture the dependencies between user achievable rates due to sharing of the wireless transmission medium [12,56,57]. In manufacturing, Andradottir, Ayhan, and Down [2] used a coupled processor model to increase the throughput of an assembly line with two stations. There, when a station empties, it helps the non-empty station to decrease its processing time.

In this work, we focus on the analysis of a retrial queueing system with two independent exogenous Poisson streams flowing into a common buffer that can hold at most one job. If a type- $i$ job finds the server busy, it is routed to a separate retrial (orbit) queue from which jobs are re-transmitted at an exponential rate and under constant retrial policy. Contrary to the model in [7], we assume that the re-transmission rate of an orbit queue, depends on the presence/absence of jobs in the other one.

Retrial queues have been extensively studied in the past (e.g., [3-5,25]). Single class retrial systems under constant retrial policy were investigated in $[6,8,21,22,24,27,28]$ (not exhaustive list). The important feature of this work is the two-class setting under constant retrial policy with coupled retrial rates. Clearly, there have been very limited results in retrial queueing literature with multiple classes of retrial customers. A two-class retrial system with arbitrary distributed service requirements and classical retrial policy was firstly analyzed in [40], whereas the extension to an arbitrary number of retrial customers was investigated in [26]. Moutzoukis and Langaris [44] introduced a non-preemptive priority mechanism in the $[26,40]$, while more recently, Langaris and Dimitriou [41] investigated a multiclass retrial queue with many phases of service. In all the above-mentioned works, a classical retrial policy was used and the authors derived expressions for the expected number of customers in orbit queues (see also [34]).

In our model, we allow the rate of re-transmission of an orbit queue to depend on the presence/absence of jobs in the other orbit queue, and using generating function analysis, we conclude in an even general functional equation than in [7]. More importantly, we investigate the mathematical analysis both for exponential and for arbitrary distributed service requirements, since the methodological approach that we use for each case is different. In particular, in the former case, the system is described by a three-dimensional Markov process, while in the latter one, we consider a two-dimensional Markov chain (MC) at service completion instants. For the model with exponential service requirements, we also provide a random walk approach using a censored MC. Indeed, our model can be seen 
as a random walk in the quarter plane modulated by a two-state MC. Using the special structure of the model we can transform it to a usual random walk in the quarter plane and investigate stability conditions. The joint orbit queue length distribution is derived by solving a homogeneous Riemann-Hilbert boundary value problem, whereas under certain assumptions on the values of the re-dispatch rates this problem reduces a Dirichlet boundary value problem. We also apply the kernel method, developed in [43], and provide the exact tail asymptotic properties for the stationary joint probability distribution of the two orbits with either an idle or a busy server. Then, we turn our attention to the analysis of the model with arbitrary distributed service requirements by investigating the joint orbit queue length distribution at service completion epochs. Using generating function analysis, we conclude in a functional equation and based on the approach developed in Cohen and Boxma [17], we derive the joint orbit queue length distribution at service completion epochs as the solution of a non-homogeneous Riemann boundary value problem.

Potential applications of our model may be found in cooperative wireless systems (i.e., network-layer cooperation), in which each source user increases its quality of service via cooperation with other users that "share" the antennas of their devices and assist the source users to transmit their data to a destination node; for example, [37,48,49]. This is so-called cooperation with relaying, and the assistant users are called relay nodes. If a transmission of a user's packet to the destination fails, the relay nodes (i.e., orbits in the retrial terminology) store the blocked packet in their buffers and try to re-transmit it to the destination later. Due to the wireless interference, the re-transmission rate of a relay node may be affected by the state of the other relay node. Other applications may arise in the modeling of local area computer networks with bus architecture $[7,38-40,46,53]$.

The contribution of our work is mainly theoretically oriented, since we provide for the first time in the related literature, a complete analysis of a model that unifies two fundamental queueing systems: the retrial queue with two orbits and constant retrial policy, and the model with coupled processors, which are both notoriously hard to study analytically. We show that the powerful and quite technical boundary value theory is an adequate technique to handle such an intricate model. An effective way to provide exact tail asymptotic results is also given.

The rest of the paper is outlined as follows. In Section 2, we describe in detail the mathematical model and derive the balance equations that are used to form the fundamental functional equation. By assuming exponential service requirements, Section 3 is devoted to the derivation of several preliminary results that are necessary for the following analysis. Necessary and sufficient conditions for the ergodicity of our system are investigated by using results from the theory of random walk in the quarter plane. Some results on the zero pairs of the kernel of the fundamental functional equation are also derived. Using that equation, and by distinguishing the analysis according to the values of re-dispatch rates of the orbits, we derive the probability generating functions of the joint orbit queue length distribution for both a busy and an idle server by formulating and solving two boundary value problems (a Riemann-Hilbert and a Dirichlet boundary value problem) in Section 4. Performance metrics are also obtained. Exact tail asymptotic properties of the stationary joint probability distribution of the two orbits for both a busy and an idle server are investigated in Section 5. Section 6 is devoted to the analysis of the model with arbitrary distributed service requirements. We prove that the joint orbit queue length distribution at departure epochs can be obtained as a solution of a non-homogeneous Riemann boundary value problem. Performance metrics are obtained and computational issues are discussed. Finally, in Section 7, a simple numerical example is presented to give an insight to the system's performance. 


\section{MODEL}

Consider a single server system accepting two types of customers, where its service station can handle at most one customer. A type $i$ customer, $i=1,2$, arrive according to a Poisson process with rate $\lambda_{i}$ and if it finds the server busy, it is blocked and routed to a separate type $i$ orbit queue of infinite capacity. Otherwise, it occupies the server immediately. Customers from the orbits try to access the server according to the constant retrial policy (see Figure 1).

We consider coupled orbit queues in the sense that when both orbit queues are nonempty, the orbit queue $i$ tries to re-dispatch a blocked customer of type $i$ to the main service station after an exponentially distributed time with rate $\mu_{i}$. If orbit queue 1 (respectively 2 ) empties, then the orbit queue 2 (respectively 1 ) changes its re-dispatch rate from $\mu_{2}$ (respectively $\left.\mu_{1}\right)$ to $\mu_{2}^{*}\left(\right.$ respectively $\left.\mu_{1}^{*}\right)$. The requested service time is independent of the type of customer, and it is exponentially distributed with rate $\mu$.

Let $N_{i}(t)$ be the number of customers in the orbit queue $i$, and $C(t)$, be the state of the server at time $t$, respectively. Assume that $C(t)=0,1$ if the server is idle and busy, respectively. Then $Q(t)=\left\{\left(N_{1}(t), N_{2}(t), C(t)\right) ; t \geq 0\right\}$ is an irreducible aperiodic Continuous Time Markov chain (CTMC) with state space $E=\{0,1, \ldots\} \times\{0,1, \ldots\} \times\{0,1\}$. Define its stationary probabilities for $i, j=0,1, \ldots, n=0,1$,

$$
p_{i, j}(n)=\lim _{t \rightarrow \infty} P\left(N_{1}(t)=i, N_{2}(t)=j, C(t)=n\right) .
$$

Then, we can write the following sets of balance equations:

1. $n=0$

$$
\begin{aligned}
p_{0,0}(0) \lambda & =\mu p_{0,0}(1), & & i=j=0, \\
p_{0, j}(0)\left[\lambda+\mu_{2}^{*}\right] & =\mu p_{0, j}(1), & & i=0, j>0, \\
p_{i, 0}(0)\left[\lambda+\mu_{1}^{*}\right] & =\mu p_{i, 0}(1), & & i>0, j=0, \\
p_{i, j}(0)\left[\lambda+\mu_{1}+\mu_{2}\right] & =\mu p_{i, j}(1), & & i>0, j>0,
\end{aligned}
$$

2. $n=1$

$$
p_{0,0}(1)[\lambda+\mu]=\lambda p_{0,0}(0)+\mu_{1}^{*} p_{1,0}(0)+\mu_{2}^{*} p_{0,1}(0), \quad i=j=0,
$$

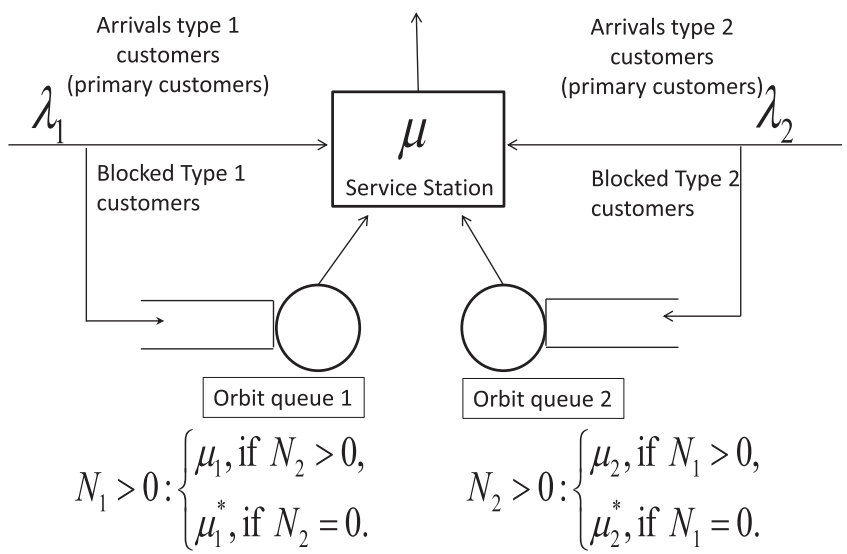

Figure 1. The model. 


$$
\begin{aligned}
p_{0, j}(1)[\lambda+\mu]= & \lambda p_{0, j}(0)+\lambda_{2} p_{0, j-1}(1)+\mu_{1} p_{1, j}(0) \\
& +\mu_{2}^{*} p_{0, j+1}(0), \quad i=0, j>0, \\
p_{i, 0}(1)[\lambda+\mu]= & \lambda p_{i, 0}(0)+\lambda_{1} p_{i-1,0}(1)+\mu_{2} p_{i, 1}(0) \\
& +\mu_{1}^{*} p_{i+1,0}(0), \quad i>0, j=0, \\
p_{i, j}(1)[\lambda+\mu]= & \lambda p_{i, j}(0)+\lambda_{1} p_{i-1, j}(1)+\lambda_{2} p_{i, j-1}(1) \\
& +\mu_{1} p_{i+1, j}(0)+\mu_{2} p_{i, j+1}(0), \quad i>0, j>0,
\end{aligned}
$$

where $\lambda=\lambda_{1}+\lambda_{2}$. Denote for $|x| \leq 1,|y| \leq 1$,

$$
\begin{aligned}
G_{i}^{(n)}(y) & =\sum_{j=0}^{\infty} p_{i, j}(n) y^{j}, i=0,1, \ldots, n=0,1, \\
H^{(n)}(x, y) & =\sum_{i=0}^{\infty} G_{i}^{(n)}(y) x^{i}=\sum_{i=0}^{\infty} \sum_{j=0}^{\infty} p_{i, j}(n) x^{i} y^{j}, \quad n=0,1 .
\end{aligned}
$$

Forming the generating functions, Eq. (2.1), becomes

$$
\begin{aligned}
{\left[\lambda+\mu_{2}^{*}\right] G_{0}^{(0)}(y)-\mu G_{0}^{(1)}(y) } & =\mu_{2}^{*} p_{0,0}(0), \quad i=0, \\
\alpha G_{i}^{(0)}(y)-\mu G_{i}^{(1)}(y) & =\left[d_{1}+\mu_{2}\right] p_{i, 0}(0), \quad i>0,
\end{aligned}
$$

where $\alpha=\lambda+\mu_{1}+\mu_{2}, d_{i}=\mu_{i}-\mu_{i}^{*}, i=1,2$.

Similarly, Eq. (2.2) becomes

$$
\begin{aligned}
{\left[\lambda+\mu-\lambda_{2} y\right] G_{0}^{(1)}(y)=} & \left(\lambda+\frac{\mu_{2}^{*}}{y}\right) G_{0}^{(0)}(y)-d_{1} p_{1,0}(0)-\frac{\mu_{2}^{*}}{y} p_{0,0}(0)+\mu_{1} G_{1}^{(0)}(y), \quad i=0 \\
{\left[\lambda+\mu-\lambda_{2} y\right] G_{i}^{(1)}(y)=} & \left(\lambda+\frac{\mu_{2}}{y}\right) G_{i}^{(0)}(y)+\lambda_{1} G_{i-1}^{(1)}(y)-d_{1} p_{i+1,0}(0) \\
& +\mu_{1} G_{i+1}^{(0)}(y)-\frac{\mu_{2}}{y} p_{i, 0}(0), \quad i>0
\end{aligned}
$$

Using (2.4) and (2.5) we arrive respectively in,

$$
\begin{aligned}
& \alpha H^{(0)}(x, y)-\mu H^{(1)}(x, y)=\left(d_{1}+\mu_{2}\right) H^{(0)}(x, 0)+\left(d_{2}+\mu_{1}\right) H^{(0)}(0, y) \\
& \quad-\left(d_{1}+d_{2}\right) H^{(0)}(0,0), \\
& \left(\lambda x y+\mu_{1} y+\mu_{2} x\right) H^{(0)}(x, y)-x y\left(\lambda_{1}(1-x)+\lambda_{2}(1-y)+\mu\right) H^{(1)}(x, y) \\
& \quad=\left(d_{1} y+\mu_{2} x\right) H^{(0)}(x, 0)+\left(d_{2} x+\mu_{1} y\right) H^{(0)}(0, y)-\left(d_{1} y+d_{2} x\right) H^{(0)}(0,0) .
\end{aligned}
$$

Equations (3.5) and (3.6) provide a relationship between generating functions for an idle and a busy server. If $H(x, y)=\left(H^{(0)}(x, y), H^{(1)}(x, y)\right)$, equations (2.6) and (2.7) in matrix 
form give

$$
H(x, y) T(x, y)=H(x, 0) T_{1}(x, y)+H(0, y) T_{2}(x, y)+H(0,0) T_{3}(x, y)
$$

where

$$
\begin{aligned}
& T(x, y)=\left(\begin{array}{cc}
\alpha & \lambda x y+\mu_{1} y+\mu_{2} x \\
\mu & -x y\left(\lambda_{1}(1-x)+\lambda_{2}(1-y)+\mu\right)
\end{array}\right), \\
& T_{1}(x, y)=\left(\begin{array}{cc}
d_{1}+\mu_{2} & d_{1} y+\mu_{2} x \\
0 & 0
\end{array}\right), \\
& T_{2}(x, y)=\left(\begin{array}{cc}
d_{2}+\mu_{1} & d_{2} x+\mu_{1} y \\
0 & 0
\end{array}\right), \\
& T_{3}(x, y)=\left(\begin{array}{cc}
-\left(d_{1}+d_{2}\right) & -\left(d_{1} y+d_{2} x\right) \\
0 & 0
\end{array}\right) .
\end{aligned}
$$

Equation (2.8) has the same form as Eq. (1.2) in the seminal paper [29]. However, in our case we deal with a matrix fundamental equation. Clearly, our model is not a random walk in the quarter plane (RWQP) as the one in [29], but it can be seen as a RWQP modulated by a two-state Markov process. Due to its special structure, (2.8) can be converted to a scalar fundamental form, which can be solved in terms of a Riemann-Hilbert boundary value problem. Indeed, solving (2.6) with respect to $H^{(1)}(x, y)$ and substituting into (2.7) we arrive in the following functional equation:

$$
K(x, y) H^{(0)}(x, y)=A(x, y) H^{(0)}(x, 0)+B(x, y) H^{(0)}(0, y)+C(x, y) H^{(0)}(0,0),
$$

where

$$
K(x, y)=\lambda_{1} \alpha(1-x) x y+\lambda_{2} \alpha(1-y) x y-\mu \mu_{1}(1-x) y-\mu \mu_{2}(1-y) x,
$$

and

$$
\begin{aligned}
& A(x, y)=d_{1} y\left[\left(\lambda_{1} x-\mu\right)(1-x)+\lambda_{2} x(1-y)\right]+\mu_{2} x\left[\lambda_{1} y(1-x)+\left(\lambda_{2} y-\mu\right)(1-y)\right], \\
& B(x, y)=d_{2} x\left[\lambda_{1} y(1-x)+\left(\lambda_{2} y-\mu\right)(1-y)\right]+\mu_{1} y\left[\left(\lambda_{1} x-\mu\right)(1-x)+\lambda_{2} x(1-y)\right], \\
& C(x, y)=-d_{1} y\left[\left(\lambda_{1} x-\mu\right)(1-x)+\lambda_{2} x(1-y)\right]-d_{2} x\left[\lambda_{1} y(1-x)+\left(\lambda_{2} y-\mu\right)(1-y)\right] .
\end{aligned}
$$

\section{GENERAL RESULTS}

Before proceeding with the main analysis, some interesting results can be obtained by the functional equation and some side conditions using the local balance approach. Denote,

$$
p_{i, .}(n)=\sum_{j=0}^{\infty} p_{i, j}(n), \quad i=0,1, \ldots, p_{., j}(n)=\sum_{i=0}^{\infty} p_{i, j}(n), \quad j=0,1, \ldots, n=0,1 .
$$

LEMMA 1:

$$
H^{(1)}(1,1)=\frac{\lambda}{\mu}
$$


1. If $\mu_{1} \mu_{2} \neq d_{1} d_{2}$,

$$
\begin{aligned}
& H^{(0)}(0,1)=\frac{\mu_{1} \mu_{2}\left[1-\frac{\lambda}{\mu}\left(\frac{\lambda_{1}+\mu_{1}}{\mu_{1}}\right)-\frac{d_{1}}{\mu_{1}}\left(1-\frac{\lambda}{\mu}\left(\frac{\lambda_{2}+\mu_{2}}{\mu_{2}}\right)\right)\right]-d_{1}\left(d_{2}-\mu_{2}\right) H^{(0)}(0,0)}{\mu_{1} \mu_{2}-d_{1} d_{2}}, \\
& H^{(0)}(1,0)=\frac{\mu_{1} \mu_{2}\left[1-\frac{\lambda}{\mu}\left(\frac{\lambda_{2}+\mu_{2}}{\mu_{2}}\right)-\frac{d_{2}}{\mu_{2}}\left(1-\frac{\lambda}{\mu}\left(\frac{\lambda_{1}+\mu_{1}}{\mu_{1}}\right)\right)\right]-d_{2}\left(d_{1}-\mu_{1}\right) H^{(0)}(0,0)}{\mu_{1} \mu_{2}-d_{1} d_{2}} .
\end{aligned}
$$

2. If $\mu_{1} \mu_{2}=d_{1} d_{2}$, then $\mu_{1}=\xi \mu_{1}^{*}, \mu_{2}=(1-\xi) \mu_{2}^{*}, 0 \leq \xi \leq 1$ and

$$
H^{(0)}(0,0)=1-\frac{\lambda}{\mu}\left[\frac{\lambda_{1}+\xi \mu_{1}^{*}}{\mu_{1}^{*}}+\frac{\lambda_{2}+(1-\xi) \mu_{2}^{*}}{\mu_{2}^{*}}\right] .
$$

Proof: For each $i=0,1, \ldots$, we consider the vertical cut between the states $\left\{N_{1}=i, C=1\right\}$ and $\left\{N_{1}=i+1, C=0\right\}$. Then,

$$
\begin{aligned}
\lambda_{1} p_{i, .}(1) & =\mu_{1}^{*} p_{i+1,0}(0)+\mu_{1} \sum_{j=1}^{\infty} p_{i+1, j}(0) \\
& =\mu_{1} p_{i+1, .}(0)-d_{1} p_{i+1,0}(0)
\end{aligned}
$$

Summing for all $i=0,1, \ldots$, we derive

$$
\begin{aligned}
\lambda_{1} H^{(1)}(1,1) & =\mu_{1}\left[H^{(0)}(1,1)-p_{0, .}(0)\right]-d_{1}\left[p_{., 0}(0)-p_{0,0}(0)\right] \\
& =\mu_{1}\left[H^{(0)}(1,1)-H^{(0)}(0,1)\right]-d_{1}\left[H^{(0)}(1,0)-H^{(0)}(0,0)\right],
\end{aligned}
$$

which in turn implies after a rearrangement and using the fact that $H^{(1)}(1,1)+$ $H^{(0)}(1,1)=1$,

$$
\left(\lambda_{1}+\mu_{1}\right) H^{(0)}(1,1)-\mu_{1} H^{(0)}(0,1)-d_{1} H^{(0)}(1,0)+d_{1} H^{(0)}(0,0)=\lambda_{1} .
$$

Similarly, by repeating the same procedure,

$$
\left(\lambda_{2}+\mu_{2}\right) H^{(0)}(1,1)-d_{2} H^{(0)}(0,1)-\mu_{2} H^{(0)}(1,0)+d_{2} H^{(0)}(0,0)=\lambda_{2} .
$$

Summing (3.5), (3.6) and subtracting the sum from (2.6), taking into account that $H^{(1)}(1,1)+H^{(0)}(1,1)=1$, we obtain $H^{(0)}(1,1)=1-\lambda / \mu$, and as result $H^{(1)}(1,1)=\lambda / \mu$. Clearly, $\lambda / \mu \leq 1$. We have to note that equations (3.5), (3.6) are "conservation of flow" relations:

$$
\begin{aligned}
\frac{\lambda_{1} \lambda}{\mu}= & \mu_{1}\left[H^{(0)}(1,1)-H^{(0)}(0,1)-H^{(0)}(1,0)+H^{(0)}(0,0)\right] \\
& +\mu_{1}^{*}\left[H^{(0)}(1,0)-H^{(0)}(0,0)\right] \\
\frac{\lambda_{2} \lambda}{\mu}= & \mu_{2}\left[H^{(0)}(1,1)-H^{(0)}(1,0)-H^{(0)}(1,0)+H^{(0)}(0,0)\right] \\
& +\mu_{2}^{*}\left[H^{(0)}(1,0)-H^{(0)}(0,0)\right] .
\end{aligned}
$$

Indeed, the first in (3.7) equates the flow of jobs into orbit queue 1, with flow of jobs out of orbit 1 . Note that, $\left(\lambda_{i} \lambda\right) / \mu$ is the throughput of jobs in orbit queue $i, i=1,2$, 
$H^{(0)}(1,1)-H^{(0)}(0,1)-H^{(0)}(1,0)+H^{(0)}(0,0)$ is the fraction of time both orbit queues are non-empty and the server is idle, while $H^{(0)}(1,0)-H^{(0)}(0,0)$, (resp. $\left.H^{(0)}(0,1)-H^{(0)}(0,0)\right)$ is the fraction of time orbit queue 1 (resp. 2) is the only non-empty and the server is idle. Substituting $H^{(0)}(1,1)=1-\lambda / \mu$ into (3.5) and (3.6) respectively we conclude that,

$$
\begin{aligned}
& H^{(0)}(0,1)=1-\frac{\lambda}{\mu}\left(\frac{\lambda_{1}+\mu_{1}}{\mu_{1}}\right)-\frac{d_{1}}{\mu_{1}}\left[H^{(0)}(1,0)-H^{(0)}(0,0)\right], \\
& H^{(0)}(1,0)=1-\frac{\lambda}{\mu}\left(\frac{\lambda_{2}+\mu_{2}}{\mu_{2}}\right)-\frac{d_{2}}{\mu_{2}}\left[H^{(0)}(0,1)-H^{(0)}(0,0)\right] .
\end{aligned}
$$

Note that $H^{(0)}(1,0)-H^{(0)}(0,0)$ is the portion of time the server is idle, the orbit queue 1 is non-empty, and the orbit queue 2 is empty. Similarly, $H^{(0)}(0,1)-H^{(0)}(0,0)$ is the portion of time the server is idle, the orbit queue 2 is the only non-empty orbit queue. Since $H^{(0)}(0,1), H^{(0)}(1,0)$ must be positive we realize that definitely,

$$
\frac{\lambda}{\mu}\left(\frac{\lambda_{1}+\mu_{1}}{\mu_{1}}\right)<1, \quad \frac{\lambda}{\mu}\left(\frac{\lambda_{2}+\mu_{2}}{\mu_{2}}\right)<1
$$

as also shown in [7]. Note that these conditions ensured stability to the model in [7]. However in our case, we need a stronger condition. To conclude, these conditions implies that $\lambda_{1} \alpha<$ $\mu \mu_{1}$ or $\lambda_{2} \alpha<\mu \mu_{2}$.

In the rest of the paper (unless other specified) we will assume that $\lambda_{1} \alpha<\mu \mu_{1}$, or equivalently $\widehat{\lambda}_{1}<\widehat{\mu}_{1}$, where $\widehat{\lambda}_{i}=\lambda_{i} \alpha, \widehat{\mu}_{i}=\mu \mu_{i}, i=1,2, \widehat{\lambda}=\widehat{\lambda}_{1}+\widehat{\lambda}_{2}$. We proceed with the proof of the Lemma by substituting (3.9) into (3.8) and derive

$H^{(0)}(0,1)\left[1-\frac{d_{1} d_{2}}{\mu_{1} \mu_{2}}\right]=1-\frac{\lambda}{\mu}\left(\frac{\lambda_{1}+\mu_{1}}{\mu_{1}}\right)-\frac{d_{1}}{\mu_{1}}\left[1-\frac{\lambda}{\mu}\left(\frac{\lambda_{2}+\mu_{2}}{\mu_{2}}\right)+\left(\frac{d_{2}}{\mu_{2}}-1\right) H^{(0)}(0,0)\right]$.

If $\mu_{1} \mu_{2} \neq d_{1} d_{2}$ we derive the first in (3.1). Similarly we can get the second.

On the other hand, if $\mu_{1} \mu_{2}=d_{1} d_{2}$ then $\frac{\mu_{1}}{\mu_{1}^{*}}+\frac{\mu_{2}}{\mu_{2}^{*}}=1$, or equivalently $\mu_{1}=\xi \mu_{1}^{*}, \mu_{2}=$ $(1-\xi) \mu_{2}^{*}, 0 \leq \xi \leq 1$. In such a case, the left-hand side (1.h.s.) of (3.10) vanishes and we can obtain (3.2). From this, we immediately deduce that

$$
\rho=\frac{\lambda}{\mu}\left[\frac{\lambda_{1}+\xi \mu_{1}^{*}}{\mu_{1}^{*}}+\frac{\lambda_{2}+(1-\xi) \mu_{2}^{*}}{\mu_{2}^{*}}\right]<1,
$$

is a necessary condition for the stability of the system if $\mu_{1} \mu_{2}=d_{1} d_{2}$. This is equivalent with,

$$
\frac{\lambda}{\mu}\left(\frac{\lambda_{1}+\xi \mu_{1}^{*}}{\xi \mu_{1}^{*}}\right)<1 \text {, and } \frac{\lambda}{\mu}\left(\frac{\lambda_{2}+(1-\xi) \mu_{2}^{*}}{(1-\xi) \mu_{2}^{*}}\right)<1 .
$$

Moreover, (3.11) yields

$$
\rho=\frac{\lambda}{\mu}\left[\frac{\lambda_{1}}{\mu_{1}^{*}}+\frac{\lambda_{2}+\mu_{2}^{*}}{\mu_{2}^{*}}\right]<1
$$

or

$$
\rho=\frac{\lambda}{\mu}\left[\frac{\lambda_{1}+\mu_{1}^{*}}{\mu_{1}^{*}}+\frac{\lambda_{2}}{\mu_{2}^{*}}\right]<1 .
$$

From hereon we will assume that this condition (3.11) (or equivalently (3.12) or (3.13) or $(3.14))$ is satisfied when $\mu_{1} \mu_{2}=d_{1} d_{2}$. 


\subsection{A Random Walk Approach and Stability Condition}

As indicated above, our model it is seen as a RWQP modulated by a finite two-state Markov process. In the following, using the special structure of our model we will convert it, using the approach in [52], to a usual RWQP [30] and investigate its stability condition.

This approach starts by considering the corresponding discrete-time MC through a uniformization technique. Without loss of generality we assume that $\theta=\lambda+\mu+\mu_{1}+\mu_{2}=$ 1. Then, we partition the transition matrix $\mathbf{P}$ of the uniformized MC according to the server state, and then consider the censored MC to the set of states of a busy server. We focus on the set of states with a busy server, because this censored MC can be explicitly expressed. We focus on the set of states of a busy server since the censored MC to the idle state does not have an explicit expression for its transition matrix.

Let $Q(k)=\left\{\left(N_{1 k}, N_{2 k}, C_{k}\right)\right\}, \quad\{0,1, \ldots\} \times\{0,1, \ldots\} \times\{0,1\}$, and $E=\{0,1, \ldots\} \times$ $\{0,1, \ldots\} \times\{1\}, E^{c}=\{0,1, \ldots\} \times\{0,1, \ldots\} \times\{0\}$. Then

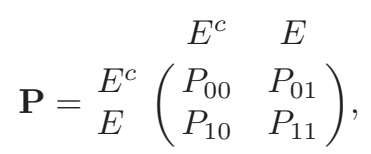

where using the lexicographical ordering for the states of $\left(N_{1 k}, N_{2 k}\right)$ :

$$
\begin{aligned}
& P_{00}=\operatorname{diag}\left(A_{0}, A_{1}, A_{1} \ldots\right), \quad P_{10}=\operatorname{diag}\left(C_{0}, C_{0}, \ldots\right), \\
& P_{01}=\left(\begin{array}{ccc}
B_{0}^{*} & & \\
B_{1} & B_{0} & \\
& B_{1} & B_{0}
\end{array}\right), \quad P_{11}=\left(\begin{array}{cccc}
D_{0} & D_{1} & & \\
& D_{0} & D_{1} & \\
& & D_{0} & D_{1}
\end{array}\right) \text {, }
\end{aligned}
$$

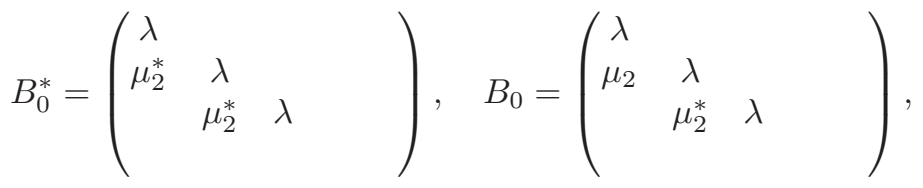

$$
\begin{aligned}
& A_{0}=\operatorname{diag}\left(\mu+\mu_{1}+\mu_{2}, 1-\left(\lambda+\mu_{2}^{*}\right), 1-\left(\lambda+\mu_{2}^{*}\right), \ldots\right) \text {, } \\
& A_{1}=\operatorname{diag}\left(1-\left(\lambda+\mu_{1}^{*}\right), \mu, \mu, \ldots\right) \\
& B_{1}=\operatorname{diag}\left(\mu_{1}^{*}, \mu_{1}, \mu_{1}, \ldots\right) \text {, } \\
& C_{0}=\mu I \text {, } \\
& D_{0}=\left(\begin{array}{ccc}
\mu_{1}+\mu_{2} & \lambda_{2} & \\
& \mu_{1}+\mu_{2} & \lambda_{2} \\
& &
\end{array}\right), \quad D_{1}=\lambda_{1} I .
\end{aligned}
$$

Since $P_{00}$ is diagonal, it is easy to have the fundamental matrix of $P_{00}$ as:

$$
\widehat{P}_{00}=\sum_{j=0}^{\infty} P_{00}^{j}=\operatorname{diag}\left(\widehat{A}_{0}, \widehat{A}_{1}, \widehat{A}_{1}, \ldots\right),
$$

where

$$
\widehat{A}_{0}=\operatorname{diag}\left(\frac{1}{\lambda}, \frac{1}{\lambda+\mu_{2}^{*}}, \frac{1}{\lambda+\mu_{2}^{*}}, \ldots\right)
$$




$$
\widehat{A}_{1}=\operatorname{diag}\left(\frac{1}{\lambda+\mu_{1}^{*}}, \frac{1}{\lambda+\mu_{1}+\mu_{2}}, \frac{1}{\lambda+\mu_{1}+\mu_{2}}, \ldots\right)
$$

The transition matrix of the censored MC to $E$ can be easily computed as:

$$
\begin{aligned}
P^{(E)}= & P_{11}+P_{10} \widehat{P}_{00} P_{01} \\
= & \left(\begin{array}{cccc}
D_{0}+\mu \widehat{A}_{0} B_{0}^{*} & D_{1} & & \\
\mu \widehat{A}_{1} B_{1} & D_{0}+\mu \widehat{A}_{0} B_{0} & D_{1} & \\
& \mu \widehat{A}_{1} B_{1} & D_{0}+\mu \widehat{A}_{0} B_{0} & D_{1} \\
& \ddots & \ddots & \ddots
\end{array}\right) .
\end{aligned}
$$

This is a simple RWQP whose one-step transition probabilities from state $(m, n)$ to $(m+i, n+j)$ are for $-1 \leq i, j \leq 1$ :

$$
\begin{aligned}
p_{(m, n ; m+i, n+j)}=\left\{\begin{array}{l}
p_{i, j} \quad \text { if } m, n>0, \\
p_{i, j}^{(1)} \quad \text { if } m>0, n=0, \\
p_{i, j}^{(2)} \quad \text { if } m=0, n>0, \\
p_{i, j}^{(0)} \quad \text { if } m=0, n=0,
\end{array}\right. \\
p_{-1,0}^{(1)}=\frac{\mu \mu_{1}^{*}}{\lambda+\mu_{1}^{*}}, \quad p_{0,0}^{(1)}=\mu_{1}+\mu_{2}+\frac{\lambda \mu}{\lambda+\mu_{1}^{*}}, \quad p_{1,0}^{(1)}=\lambda_{1}, \quad p_{0,1}^{(1)}=\lambda_{2}, \\
p_{0,-1}^{(2)}=\frac{\mu \mu_{2}^{*}}{\lambda+\mu_{2}^{*}}, \quad p_{0,0}^{(2)}=\mu_{1}+\mu_{2}+\frac{\lambda \mu}{\lambda+\mu_{2}^{*}}, \quad p_{1,0}^{(2)}=\lambda_{1}, \quad p_{0,1}^{(2)}=\lambda_{2}, \\
p_{0,0}^{(0)}=\mu_{1}+\mu_{2}+\mu, \quad p_{1,0}^{(0)}=\lambda_{1}, \quad p_{0,1}^{(0)}=\lambda_{2}, \\
p_{-1,0}=\frac{\mu \mu_{1}}{\lambda+\mu_{1}+\mu_{2}}, \quad p_{0,0}=\mu_{1}+\mu_{2}+\frac{\lambda \mu}{\lambda+\mu_{1}+\mu_{2}}, \quad p_{1,0}=\lambda_{1}, \\
p_{0,1}=\lambda_{2}, \quad p_{0,-1}=\frac{\mu \mu_{2}}{\lambda+\mu_{1}+\mu_{2}} .
\end{aligned}
$$

Using the notation in Fayolle, Iasnogorodski, and Malyshev [30], and define for $|x| \leq 1$, $|y| \leq 1, k=0,1$,

$$
\begin{aligned}
\pi^{(k)}(x, y) & :=\sum_{i=1}^{\infty} \sum_{j=1}^{\infty} p_{i, j}(k) x^{i-1} y^{j-1}, \\
\pi_{1}^{(k)}(x) & :=\pi^{(k)}(x, 0)=\sum_{i=1}^{\infty} p_{i, 0}(k) x^{i-1} \\
\pi_{2}^{(k)}(y) & :=\pi^{(k)}(0, y)=\sum_{j=1}^{\infty} p_{0, j}(k) y^{j-1} .
\end{aligned}
$$

For this censored random walk, the fundamental form (Eq. (1.3.6) in [30]) is given by:

$$
-k^{(1)}(x, y) \pi^{(1)}(x, y)=a^{(1)}(x, y) \pi_{1}^{(1)}(x)+b^{(1)}(x, y) \pi_{2}^{(1)}(y)+c^{(1)}(x, y) \pi^{(1)}(0,0),
$$


where

$$
\begin{aligned}
k^{(1)}(x, y) & =x y\left[\sum_{i=-1}^{1} \sum_{j=-1}^{1} p_{i, j} x^{i} y^{j}-1\right] \\
& =a(x) y^{2}+b(x) y+c(x) \\
& =\frac{x y}{\lambda+\mu_{1}+\mu_{2}}\left[\widehat{\lambda}_{1} x+\widehat{\lambda}_{2} y+\widehat{\mu}_{1} x^{-1}+\widehat{\mu}_{2} y^{-1}-\left(\widehat{\lambda}+\widehat{\mu}_{1}+\widehat{\mu}_{2}\right)\right] \\
a^{(1)}(x, y) & =x\left[\sum_{i=-1}^{1} \sum_{j=0}^{1} p_{i, j}^{(1)} x^{i} y^{j}-1\right]=a_{1}(x) y+b_{1}(x) \\
& =\frac{x\left[\lambda_{1}\left(\lambda+\mu_{1}^{*}\right) x+\lambda_{2}\left(\lambda+\mu_{1}^{*}\right) y+\mu \mu_{1}^{*} x^{-1}-\lambda\left(\lambda+\mu_{1}^{*}\right)-\mu \mu_{1}^{*}\right]}{\lambda+\mu_{1}^{*}} \\
b^{(1)}(x, y) & =y\left[\sum_{i=0}^{1} \sum_{j=-1}^{1} p_{i, j}^{(2)} x^{i} y^{j}-1\right] \\
& =a_{2}(x) y^{2}+b_{2}(x) y+c_{2}(x) \\
& =\frac{y\left[\lambda_{1}\left(\lambda+\mu_{2}^{*}\right) x+\lambda_{2}\left(\lambda+\mu_{2}^{*}\right) y+\mu \mu_{2}^{*} y^{-1}-\lambda\left(\lambda+\mu_{2}^{*}\right)-\mu \mu_{2}^{*}\right]}{\lambda+\mu_{2}^{*}} \\
c^{(1)}(x, y) & =\sum_{i=0} \sum_{j=0}^{1} p_{i, j}^{(0)} x^{i} y^{j}-1=a_{0}(x) y+b_{0}(x) \\
& =\lambda_{1} x+\lambda_{2} y-\lambda,
\end{aligned}
$$

where

$$
\begin{aligned}
& a(x)=p_{0,1} x, \quad b(x)=p_{-1,0}-\left(1-p_{0,0}\right) x+p_{1,0} x^{2}, \quad c(x)=p_{0,-1} x ; \\
& a_{1}(x)=p_{0,1}^{(1)} x, \quad b_{1}(x)=p_{-1,0}^{(1)}-\left(1-p_{0,0}^{(1)}\right) x+p_{1,0}^{(1)} x^{2} \\
& a_{2}(x)=p_{0,1}^{(2)}, \quad b_{2}(x)=p_{0,0}^{(2)}-1+p_{1,0}^{(2)} x, \quad c_{2}(x)=p_{0,-1}^{(2)} \\
& a_{0}(x)=p_{0,1}^{(0)} x, \quad b_{0}(x)=p_{1,0}^{(0)} x-\left(1-p_{0,0}^{(0)}\right) .
\end{aligned}
$$

Our model is now converted to a usual RWQP and we can proceed as in Fayolle et al. [30] and solve (3.17) by transform it into a Riemann-Carleman boundary value problem. As stated above, the censored chain to the idle state does not have an explicit expression for its transition matrix. However, it is easy to see that the functional Eq. (2.9) is equivalent to:

$$
\begin{aligned}
K(x, y) \pi^{(0)}(x, y)= & \frac{A(x, y)-K(x, y)}{y} \pi_{1}^{(0)}(x)+\frac{B(x, y)-K(x, y)}{x} \pi_{2}^{(0)}(y) \\
& +\frac{C(x, y)+A(x, y)+B(x, y)-K(x, y)}{x y} \pi^{(0)}(0,0) .
\end{aligned}
$$

After some algebra, the above equation can be written as:

$$
-k^{(0)}(x, y) \pi^{(0)}(x, y)=a^{(0)}(x, y) \pi_{1}^{(0)}(x)+b^{(0)}(x, y) \pi_{2}^{(0)}(y)+c^{(0)}(x, y) \pi^{(0)}(0,0),
$$


where

$$
\begin{aligned}
& k^{(0)}(x, y)=\left(\lambda+\mu_{1}+\mu_{2}\right) k^{(1)}(x, y), \\
& a^{(0)}(x, y)=\left(\lambda+\mu_{1}^{*}\right) a^{(1)}(x, y), \\
& b^{(0)}(x, y)=\left(\lambda+\mu_{2}^{*}\right) b^{(1)}(x, y), \\
& c^{(0)}(x, y)=\lambda c^{(1)}(x, y) .
\end{aligned}
$$

The functional Eq. (3.19) is the fundamental form corresponding to a RWQP defined by:

$$
\begin{aligned}
\widehat{p}_{-1,0}^{(1)} & =\mu \mu_{1}^{*}, \quad \widehat{p}_{0,0}^{(1)}=1-\left[\lambda\left(\lambda+\mu_{1}^{*}\right)+\mu \mu_{1}^{*}\right], \quad \widehat{p}_{1,0}^{(1)}=\lambda_{1}\left(\lambda+\mu_{1}^{*}\right), \\
\widehat{p}_{0,1}^{(1)} & =\lambda_{2}\left(\lambda+\mu_{1}^{*}\right), \\
\widehat{p}_{0,-1}^{(2)} & =\mu \mu_{2}^{*}, \quad \widehat{p}_{0,0}^{(2)}=1-\left[\lambda\left(\lambda+\mu_{2}^{*}\right)+\mu \mu_{2}^{*}\right], \quad \widehat{p}_{1,0}^{(2)}=\lambda_{1}\left(\lambda+\mu_{2}^{*}\right), \\
\widehat{p}_{0,1}^{(2)} & =\lambda_{2}\left(\lambda+\mu_{2}^{*}\right), \\
\widehat{p}_{0,0}^{(0)} & =1-\lambda^{2}, \quad \widehat{p}_{1,0}^{(0)}=\lambda \lambda_{1}, \quad \widehat{p}_{0,1}^{(0)}=\lambda \lambda_{2}, \\
\widehat{p}_{-1,0} & =\widehat{\mu}_{1}, \quad \widehat{p}_{0,0}=1-\left(\widehat{\lambda}+\widehat{\mu}_{1}+\widehat{\mu}_{2}\right), \quad \widehat{p}_{1,0}=\widehat{\lambda}_{1}, \\
\widehat{p}_{0,1} & =\widehat{\lambda}_{2}, \quad \widehat{p}_{0,-1}=\widehat{\mu}_{2} .
\end{aligned}
$$

Set

$$
\left\{\begin{array}{l}
M=\left(M_{x}, M_{y}\right)=\left(\sum i \widehat{p}_{i, j}, \sum j \widehat{p}_{i, j}\right)=\left(\widehat{\lambda}_{1}-\widehat{\mu}_{1}, \widehat{\lambda}_{2}-\widehat{\mu}_{2}\right), \\
M^{(1)}=\left(M_{x}^{(1)}, M_{y}^{(1)}\right)=\left(\sum i \widehat{p}_{i, j}^{(1)}, \sum j \widehat{p}_{i, j}^{(1)}\right)=\left(\lambda_{1}\left(\lambda+\mu_{1}^{*}\right)-\mu \mu_{1}^{*}, \lambda_{2}\left(\lambda+\mu_{1}^{*}\right)\right), \\
M^{(2)}=\left(M_{x}^{(2)}, M_{y}^{(2)}\right)=\left(\sum i \widehat{p}_{i, j}^{(2)}, \sum j \widehat{p}_{i, j}^{(2)}\right)=\left(\lambda_{1}\left(\lambda+\mu_{2}^{*}\right), \lambda_{2}\left(\lambda+\mu_{2}^{*}\right)-\mu \mu_{2}^{*}\right) .
\end{array}\right.
$$

The following theorem is due to Fayolle et al. [30].

THEOREM 1: When $M \neq 0$, a random walk is ergodic if, and only if, one of the following conditions holds,

1.

$$
\left\{\begin{array}{l}
M_{x}<0, M_{y}<0 \\
M_{x} M_{y}^{(1)}-M_{y} M_{x}^{(1)}<0 \\
M_{y} M_{x}^{(2)}-M_{x} M_{y}^{(2)}<0
\end{array}\right.
$$

2. $M_{x}<0, M_{y} \geq 0, M_{y} M_{x}^{(2)}-M_{x} M_{y}^{(2)}<0$;

3. $M_{x} \geq 0, M_{y}<0, M_{x} M_{y}^{(1)}-M_{y} M_{x}^{(1)}<0$.

Clearly, $M \neq 0$, since we have already assumed that $M_{x}<0$, which implies $\widehat{\lambda}_{1}<\widehat{\mu}_{1}$ (Condition 3. is also not considered here. Thus, we only focus on conditions 1. and 2.). The following theorem gives sufficient and necessary conditions for the ergodicity of the random walk that describes our system: 
THEOREM 2: Let $\widehat{\lambda}_{1}<\widehat{\mu}_{1}$. Then, the system is ergodic if and only if

1.

$$
\left.\begin{array}{l}
\mu_{1}^{*}>\frac{\lambda\left(\lambda_{1} \mu_{2}-\lambda_{2} \mu_{1}\right)}{\widehat{\mu}_{2}-\lambda\left(\lambda_{2}+\mu_{2}\right)}, \\
\mu_{2}^{*}>\frac{\lambda\left(\lambda_{2} \mu_{1}-\lambda_{1} \mu_{2}\right)}{\widehat{\mu}_{1}-\lambda\left(\lambda_{1}+\mu_{1}\right)} .
\end{array}\right\}, \quad \widehat{\lambda}_{2}<\widehat{\mu}_{2}
$$

2.

$$
\mu_{2}^{*}>\frac{\lambda\left(\lambda_{2} \mu_{1}-\lambda_{1} \mu_{2}\right)}{\widehat{\mu}_{1}-\lambda\left(\lambda_{1}+\mu_{1}\right)}, \quad \widehat{\lambda}_{2} \geq \widehat{\mu}_{2}
$$

Proof: The proof of the theorem is completely based on Theorem 1. After some algebra we can see that:

$$
M_{x} M_{y}^{(1)}-M_{y} M_{x}^{(1)}<0 \Leftrightarrow d_{1}+\mu_{2} \frac{\lambda\left(\lambda_{1}+\mu_{1}\right)-\widehat{\mu}_{1}}{\widehat{\mu}_{2}-\lambda\left(\lambda_{2}+\mu_{2}\right)}<0 \Leftrightarrow \mu_{1}^{*}>\frac{\lambda\left(\lambda_{1} \mu_{2}-\lambda_{2} \mu_{1}\right)}{\widehat{\mu}_{2}-\lambda\left(\lambda_{2}+\mu_{2}\right)} .
$$

Similarly,

$$
M_{y} M_{x}^{(2)}-M_{x} M_{y}^{(2)}<0 \Leftrightarrow d_{2}+\mu_{1} \frac{\lambda\left(\lambda_{2}+\mu_{2}\right)-\widehat{\mu}_{2}}{\widehat{\mu}_{1}-\lambda\left(\lambda_{1}+\mu_{1}\right)}<0 \Leftrightarrow \mu_{2}^{*}>\frac{\lambda\left(\lambda_{2} \mu_{1}-\lambda_{1} \mu_{2}\right)}{\widehat{\mu}_{1}-\lambda\left(\lambda_{1}+\mu_{1}\right)} .
$$

Note that this Theorem is similar to Theorem 7.2, p. 345 in [29], and will help us to determine the index of a Riemann-Hilbert problem, the solution of which will give us the joint probability generating function of the orbit queue length distribution for an idle server (see Section 4). Moreover, it is easy to see that conditions 1 and 2 in Theorem 2, reduce to condition (3.11) when $\mu_{1} \mu_{2}=d_{1} d_{2}$.

\subsection{Zero Pairs of the Kernel}

For fixed $y$, the equation $K(x, y)=0$ in variable $x$ has two roots

$$
X_{ \pm}(y)=\frac{-\left(\widehat{\lambda}_{2} y^{2}-\left(\widehat{\lambda}+\widehat{\mu}_{1}+\widehat{\mu}_{2}\right) y+\widehat{\mu}_{2}\right) \pm \sqrt{\Delta_{2}(y)}}{2 \widehat{\lambda}_{1} y}
$$

where

$$
\Delta_{2}(y)=\left(\widehat{\lambda}_{2} y^{2}-\left(\widehat{\lambda}+\widehat{\mu}_{1}+\widehat{\mu}_{2}\right) y+\widehat{\mu}_{2}\right)^{2}-4 \widehat{\lambda}_{1} \widehat{\mu}_{1} y^{2} .
$$

We can easily prove that $\Delta_{2}(y)$ has four real roots such that $0<y_{1}<y_{2} \leq 1 \leq y_{3}<y_{4}$ given by,

$$
\begin{aligned}
& y_{1}=\frac{\widehat{\lambda}+\widehat{\mu}_{1}+\widehat{\mu}_{2}+2 \sqrt{\widehat{\lambda}_{1} \widehat{\mu}_{1}}-\sqrt{s_{X}^{(1)}}}{2 \widehat{\lambda}_{2}}, \quad y_{2}=\frac{\widehat{\lambda}+\widehat{\mu}_{1}+\widehat{\mu}_{2}-2 \sqrt{\widehat{\lambda}_{1} \widehat{\mu}_{1}}-\sqrt{s_{X}^{(2)}}}{2 \widehat{\lambda}_{2}}, \\
& y_{3}=\frac{\widehat{\lambda}+\widehat{\mu}_{1}+\widehat{\mu}_{2}-2 \sqrt{\widehat{\lambda}_{1} \widehat{\mu}_{1}}+\sqrt{s_{X}^{(2)}}}{2 \widehat{\lambda}_{2}}, \quad y_{4}=\frac{\widehat{\lambda}+\widehat{\mu}_{1}+\widehat{\mu}_{2}+2 \sqrt{\widehat{\lambda}_{1} \widehat{\mu}_{1}}+\sqrt{s_{X}^{(1)}}}{2 \widehat{\lambda}_{2}},
\end{aligned}
$$

where

$$
\begin{aligned}
& s_{X}^{(1)}=\left(\widehat{\lambda}+\widehat{\mu}_{1}+\widehat{\mu}_{2}+2 \sqrt{\widehat{\lambda}_{1} \widehat{\mu}_{1}}\right)^{2}-4 \widehat{\lambda}_{2} \widehat{\mu}_{2}, \\
& s_{X}^{(2)}=\left(\widehat{\lambda}+\widehat{\mu}_{1}+\widehat{\mu}_{2}-2 \sqrt{\widehat{\lambda}_{1} \widehat{\mu}_{1}}\right)^{2}-4 \widehat{\lambda}_{2} \widehat{\mu}_{2} .
\end{aligned}
$$


It is easy to see that $y_{2}=1$ when $\widehat{\lambda}_{1}=\widehat{\mu}_{1}$ and $\widehat{\lambda}_{2} \leq \widehat{\mu}_{2}$, and $y_{3}=1$ when $\widehat{\lambda}_{1}=\widehat{\mu}_{1}$ and $\widehat{\lambda}_{2} \geq \widehat{\mu}_{2}$. Define for $y \in \mathbb{C}-\left(\left[y_{1}, y_{2}\right] \cup\left[y_{3}, y_{4}\right]\right)$ the two branches of $X(y)$ by

$$
X_{0}(y)=\left\{\begin{array}{ll}
X_{-}(y) & \text { if }\left|X_{-}(y)\right| \leq\left|X_{+}(y)\right|, \\
X_{+}(y) & \text { if }\left|X_{-}(y)\right|>\left|X_{+}(y)\right| ;
\end{array}, X_{1}(y)= \begin{cases}X_{+}(y) & \text { if }\left|X_{-}(y)\right| \leq\left|X_{+}(y)\right|, \\
X_{-}(y) & \text { if }\left|X_{-}(y)\right|>\left|X_{+}(y)\right|\end{cases}\right.
$$

The function $X_{0}(y)$ is analytic in $\mathbb{C}-\left(\left[y_{1}, y_{2}\right] \cup\left[y_{3}, y_{4}\right]\right)$ and the function $X_{1}(y)$ is meromorphic in $\mathbb{C}-\left(\left[y_{1}, y_{2}\right] \cup\left[y_{3}, y_{4}\right]\right)$ with a single pole at 0 . The function $X_{0}(y)$ analytically continues in the whole of $\mathbb{C}-\left(\left[y_{1}, y_{2}\right] \cup\left[y_{3}, y_{4}\right]\right)$ the function $X_{+}(y)$ defined for $y<y_{1}$. Similarly, the function $X_{1}(y)$ meromorphically continues in the same domain the function $X_{-}(y)$ defined for $y<y_{1}$.

Similarly, for fixed $x$, the equation $K(x, y)=0$ in variable $y$ has two roots

$$
Y_{ \pm}(x)=\frac{-\left(\widehat{\lambda}_{1} x^{2}-\left(\widehat{\lambda}+\widehat{\mu}_{1}+\widehat{\mu}_{2}\right) x+\widehat{\mu}_{1}\right) \pm \sqrt{\Delta_{1}(x)}}{2 \widehat{\lambda}_{2} x}
$$

where

$$
\Delta_{1}(x)=\left(\widehat{\lambda}_{1} x^{2}-\left(\widehat{\lambda}+\widehat{\mu}_{1}+\widehat{\mu}_{2}\right) x+\widehat{\mu}_{1}\right)^{2}-4 \widehat{\lambda}_{2} \widehat{\mu}_{2} x^{2} .
$$

The discriminant $\Delta_{1}(x)$ has four real roots such that $0<x_{1}<x_{2} \leq 1<\widehat{\mu}_{1} / \widehat{\lambda}_{1}<x_{3}<$ $x_{4}$ given by,

$$
\begin{aligned}
& x_{1}=\frac{\widehat{\lambda}+\widehat{\mu}_{1}+\widehat{\mu}_{2}+2 \sqrt{\widehat{\lambda}_{2} \widehat{\mu}_{2}}-\sqrt{s_{Y}^{(1)}}}{2 \widehat{\lambda}_{1}}, \quad x_{2}=\frac{\widehat{\lambda}+\widehat{\mu}_{1}+\widehat{\mu}_{2}-2 \sqrt{\widehat{\lambda}_{2} \widehat{\mu}_{2}}-\sqrt{s_{Y}^{(2)}}}{2 \widehat{\lambda}_{1}}, \\
& x_{3}=\frac{\widehat{\lambda}+\widehat{\mu}_{1}+\widehat{\mu}_{2}-2 \sqrt{\widehat{\lambda}_{2} \widehat{\mu}_{2}}+\sqrt{s_{Y}^{(2)}}}{2 \widehat{\lambda}_{1}}, \quad x_{4}=\frac{\widehat{\lambda}+\widehat{\mu}_{1}+\widehat{\mu}_{2}+2 \sqrt{\widehat{\lambda}_{2} \widehat{\mu}_{2}}+\sqrt{s_{Y}^{(1)}}}{2 \widehat{\lambda}_{1}},
\end{aligned}
$$

where

$$
\begin{aligned}
& s_{X}^{(1)}=\left(\widehat{\lambda}+\widehat{\mu}_{1}+\widehat{\mu}_{2}+2 \sqrt{\widehat{\lambda}_{1} \widehat{\mu}_{1}}\right)^{2}-4 \widehat{\lambda}_{2} \widehat{\mu}_{2}, \\
& s_{X}^{(2)}=\left(\widehat{\lambda}+\widehat{\mu}_{1}+\widehat{\mu}_{2}-2 \sqrt{\widehat{\lambda}_{1} \widehat{\mu}_{1}}\right)^{2}-4 \widehat{\lambda}_{2} \widehat{\mu}_{2} .
\end{aligned}
$$

Note that $x_{2}=1$ when $\widehat{\lambda}_{2}=\widehat{\mu}_{2}$ and $\widehat{\lambda}_{1} \leq \widehat{\mu}_{1}$, and $x_{3}=1$ when $\widehat{\lambda}_{2}=\widehat{\mu}_{2}$ and $\widehat{\lambda}_{1} \geq \widehat{\mu}_{1}$. The functions $Y_{ \pm}(x)$ defined in (3.23) are well defined for $x \in \mathbb{C}-\left(\left[x_{1}, x_{2}\right] \cup\left[x_{3}, x_{4}\right]\right)$, and the two branches are defined by

$$
Y_{0}(x)=\left\{\begin{array}{ll}
Y_{-}(x) & \text { if }\left|Y_{-}(x)\right| \leq\left|Y_{+}(x)\right|, \\
Y_{+}(x) & \text { if }\left|Y_{-}(x)\right|>\left|Y_{+}(x)\right| ;
\end{array}, Y_{1}(x)= \begin{cases}Y_{+}(x) & \text { if }\left|Y_{-}(x)\right| \leq\left|Y_{+}(x)\right|, \\
Y_{-}(x) & \text { if }\left|Y_{-}(x)\right|>\left|Y_{+}(x)\right| .\end{cases}\right.
$$

Similar properties as for $X_{ \pm}(y)$ also holds for $Y_{ \pm}(x)$. The following conformal mapping result is classical and for more details see [30].

Lemma 2: The function $X_{0}(y)$ is a conformal mapping from the open set $D\left(0, \sqrt{\frac{\widehat{\mu}_{2}}{\lambda_{2}}}\right)-$ $\left[y_{1}, y_{2}\right]$ onto the open set $D\left(0, \sqrt{\frac{\widehat{\mu}_{1}}{\widehat{\lambda}_{1}}}\right)-\left[x_{1}, x_{2}\right]$, where $D\left(0, \sqrt{\frac{\widehat{\mu}_{i}}{\hat{\lambda}_{i}}}\right)$ denotes the disk with center 0 and radius $\sqrt{\frac{\hat{\mu}_{i}}{\widehat{\lambda}_{i}}}, i=1,2$. The reciprocal function is $Y_{0}(x)$. 
To conclude, note that $X_{-}(1) \leq X_{+}(1)$, and $X_{-}(1)<1$ if and only if $\widehat{\lambda}_{1}>\widehat{\mu}_{1}$ and then $X_{-}(1)=\widehat{\mu}_{1} / \widehat{\lambda}_{1}$. Similarly, $Y_{-}(1)<1$ if and only if $\widehat{\lambda}_{2}>\widehat{\mu}_{2}$, in which case $Y_{-}(1)=\widehat{\mu}_{2} / \widehat{\lambda}_{2}$. For more properties on the zero pairs of the kernel the interested reader is referred to [7] (Proposition 3, p. 14), and [52] (Lemmas 4.1, 4.2, pp. 10-11).

Using similar arguments as in [29], Section III, we can prove that $H^{(0)}(x, 0)$ (resp. $\left.H^{(0)}(0, y)\right)$ can be continued as a meromorphic function to the whole complex plane cut along the segment $\left[x_{3}, x_{4}\right]$ (resp. $\left[y_{3}, y_{4}\right]$ ). In the remaining text, we assume that $\widehat{\lambda}_{1}<\widehat{\mu}_{1}$. Since the continuation of $H^{(0)}(x, 0)$ (resp. $H^{(0)}(0, y)$ ) may have poles between the unit circle and the $|x|=\sqrt{\widehat{\mu}_{1} / \widehat{\lambda}_{1}}$ (resp. $|y|=\sqrt{\widehat{\mu}_{2} / \widehat{\lambda}_{2}}$ if $\left.\widehat{\lambda}_{2}<\widehat{\mu}_{2}\right)$. However, from the analyticity of $A\left(x, Y_{0}(x)\right) H^{(0)}(x, 0)$ it follows that the poles of $H^{(0)}(x, 0)$ must coincide with the zeros of $A\left(x, Y_{0}(x)\right)$ in the band between the unit circle and $|x|=\sqrt{\widehat{\mu}_{1} / \widehat{\lambda}_{1}}$.

\subsection{Intersection Points of $K(x, y)=0$ and $A(x, y)=0$}

When $K(x, y)=0$, we can see from $(2.9)$ that we can express $H^{(0)}(x, 0)\left(\right.$ resp. $\left.H^{(0)}(0, y)\right)$ as a function of $H^{(0)}(0, y)$ (resp. $\left.H^{(0)}(x, 0)\right)$ and $C(x, y)$, where $A(x, y)$ appears in the denominator. Thus, the common solutions of the equations $K(x, y)=0$ and $A(x, y)=0$ are potential singularities for the functions $H^{(0)}(x, 0)$ and $H^{(0)}(0, y)$.

The common roots in variable $\boldsymbol{y}$. Let $y \in C-\left(\left[y_{1}, y_{2}\right] \cup\left[y_{3}, y_{4}\right]\right)$ and $K(x, y)=0, x=$ $X_{ \pm}(y)$. If in addition $A(x, y)=0, y$ is a root of the resultant in $x$ of the two polynomials $K(x, y)$ and $A(x, y)$, denoted by $Q_{x}(y) . Q_{x}(y)$ is a polynomial of degree 5 in $y$, which has at most four distinct zeros in $C$. The point 0 is a double root. Another trivial root is 1 since $K(1,1)=0$ and $A(1,1)=0$. It can be shown that the resultant $Q_{x}(y)$ can actually be decomposed as

$$
Q_{x}(y)=\lambda_{1} \mu^{2} y^{2}(y-1) \widehat{Q}_{x}(y)
$$

where

$$
\begin{aligned}
\widehat{Q}_{x}(y)= & \lambda \lambda_{2}\left(d_{1}+\mu_{2}\right)\left(\lambda d_{1}-\mu_{2} \mu_{1}^{*}\right) y^{2}+\lambda \mu_{2} y\left[\mu \mu_{1}^{*}\left(d_{1}+\mu_{2}\right)\right. \\
& \left.-\left(\lambda d_{1}-\mu_{2} \mu_{1}^{*}\right)\left(\lambda+\mu_{1}^{*}\right)\right]-\widehat{\mu}_{2} \mu_{1}^{*} \mu_{2}\left(\lambda+\mu_{1}^{*}\right) .
\end{aligned}
$$

The common roots in variable $x$. Letting now $x \in C-\left(\left[x_{1}, x_{2}\right] \cup\left[x_{3}, x_{4}\right]\right)$ and $K(x, y)=$ $0, y=Y_{ \pm}(x)$, we can easily show that the resultant in $y$ of the two polynomials $K(x, y)$ and $A(x, y)$, denoted by $P_{y}(x)$ is

$$
P_{y}(x)=\lambda_{2} \mu^{2} \mu_{2} x^{2}(x-1) \widehat{P}_{y}(x)
$$

where

$$
\begin{aligned}
\widehat{P}_{y}(x)= & \lambda \lambda_{1}\left(\lambda+\mu_{1}^{*}\right)\left(d_{1}+\mu_{2}\right) x^{2}-\lambda\left[\mu \mu_{1}^{*}\left(d_{1}+\mu_{2}\right)\right. \\
& \left.+\left(\lambda d_{1}-\mu_{2} \mu_{1}^{*}\right)\left(\lambda+\mu_{1}^{*}\right)\right] x+\mu_{1}^{*} \mu\left(\lambda d_{1}-\mu_{2} \mu_{1}^{*}\right) .
\end{aligned}
$$

The roots of this polynomial are real since the discriminant

$$
\begin{aligned}
\Delta_{y}^{(1)}= & \left(\lambda \mu_{1}^{*} \mu\left(d_{1}+\mu_{2}\right)\right)^{2}+\left(\lambda\left(\lambda d_{1}-\mu_{2} \mu_{1}^{*}\right)\left(\lambda+\mu_{1} *\right)\right)^{2} \\
& +2 \lambda\left(\lambda_{2}-\lambda_{1}\right) \mu_{1}^{*} \mu\left(d_{1}+\mu_{2}\right)\left(\lambda d_{1}-\mu_{2} \mu_{1}^{*}\right) \\
\geq & {\left[\lambda \mu_{1}^{*} \mu\left|d_{1}+\mu_{2}\right|-\lambda\left|\lambda d_{1}-\mu_{2} \mu_{1}^{*}\right|\left(\lambda+\mu_{1} *\right)\right]^{2} \geq 0, }
\end{aligned}
$$


given by

$$
\xi_{ \pm}=\frac{\lambda\left[\mu \mu_{1}^{*}\left(d_{1}+\mu_{2}\right)+\left(\lambda d_{1}-\mu_{2} \mu_{1}^{*}\right)\left(\lambda+\mu_{1}^{*}\right)\right] \pm \sqrt{\Delta_{y}^{(1)}}}{2 \lambda \lambda_{1}\left(\lambda+\mu_{1}^{*}\right)\left(d_{1}+\mu_{2}\right)} .
$$

To show that these roots are positive we follow the lines in [29], Section V, and subtract $A\left(x, Y_{0}(x)\right)$ from $K\left(x, Y_{0}(x)\right)$ to obtain,

$$
\lambda_{1}\left(\lambda+\mu_{1}^{*}\right)(1-x)+\mu \mu_{1}^{*}\left(1-\frac{1}{x}\right)+\lambda_{2}\left(\lambda+\mu_{1}^{*}\right)\left(1-Y_{0}(x)\right)=0 .
$$

Then, $x \leq 0$, yields $Y_{0}(x)>1$ which is impossible. Thus, any root is positive and cannot belong to the cut $\left[x_{1}, x_{2}\right]$ since $Y_{0}(x)$ is a complex number and $A\left(x, Y_{0}(x)\right)$ never vanishes in that case.

\section{FORMULATION OF THE BOUNDARY VALUE PROBLEMS}

In the following, we distinguish the analysis in two cases, based on certain assumptions on the values of re-transmission rates.

\subsection{Reduction to a Riemann-Hilbert Boundary Value Problem}

In the following, we assume the case where $\mu_{1} \mu_{2} \neq d_{1} d_{2}$. We can easily observe that,

$$
d_{2}\left(d_{1}-\mu_{1}\right) A(x, y)+d_{1}\left(d_{2}-\mu_{2}\right) B(x, y)=\left(\mu_{1} \mu_{2}-d_{1} d_{2}\right) C(x, y) .
$$

Using (4.1), we observe that

$$
\begin{aligned}
& \operatorname{Im}\left(\frac{C(x, y)}{B(x, y)}\right)=\frac{d_{2}\left(d_{1}-\mu_{1}\right)}{d_{1} d_{2}-\mu_{1} \mu_{2}} \operatorname{Re}\left(i \frac{A(x, y)}{B(x, y)}\right), \\
& \operatorname{Im}\left(\frac{C(x, y)}{A(x, y)}\right)=\frac{d_{1}\left(d_{2}-\mu_{2}\right)}{d_{1} d_{2}-\mu_{1} \mu_{2}} \operatorname{Re}\left(i \frac{B(x, y)}{A(x, y)}\right),
\end{aligned}
$$

leading to the following transformation:

$$
\begin{gathered}
G(x)=H^{(0)}(x, 0)-\frac{d_{2}\left(d_{1}-\mu_{1}\right)}{d_{1} d_{2}-\mu_{1} \mu_{2}} H^{(0)}(0,0), \\
W(y)=H^{(0)}(0, y)-\frac{d_{1}\left(d_{2}-\mu_{2}\right)}{d_{1} d_{2}-\mu_{1} \mu_{2}} H^{(0)}(0,0),
\end{gathered}
$$

and thus the fundamental relation (2.9) is rewritten for $(x, y)$ such that $K(x, y)=0,|x| \leq 1$, $|y| \leq 1$

$$
G(x) A(x, y)+W(y) B(x, y)=0 .
$$

Therefore, the l.h.s. of (4.3) must vanish for all pairs $\left(X_{0}(y), y\right)$ such that $|y|=1$, which yields,

$$
A\left(X_{0}(y), y\right) G\left(X_{0}(y)\right)=-B\left(X_{0}(y), y\right) W(y),|y|=1 .
$$

The right-hand side (r.h.s.) of (4.4) is analytic for $y \in D(0,1)-\left[y_{1}, y_{2}\right]$ and continuous for $|y|=1$, so it can be analytically continued up to the interval $\left[y_{1}, y_{2}\right]$, which implies that for 
$y \in\left[y_{1}, y_{2}\right]$

$$
A\left(X_{0}(y), y\right) G\left(X_{0}(y)\right)=-B\left(X_{0}(y), y\right) W(y) .
$$

We proved in Lemma 7 that $B\left(X_{0}(y), y\right) \neq 0$ for $y \in\left[y_{1}, y_{2}\right]$ (similarly we also can prove that $A\left(X_{0}(y), y\right) \neq 0$ for $\left.y \in\left[y_{1}, y_{2}\right]\right)$. Let $X_{0}(y)=x$, so that $Y_{0}\left(X_{0}(y)\right)=y$, we may write:

$$
A\left(x, Y_{0}(x)\right) G(x)=-B\left(x, Y_{0}(x)\right) W\left(Y_{0}(x)\right), \quad|x|=\sqrt{\frac{\widehat{\mu}_{1}}{\widehat{\lambda}_{1}}} .
$$

Following [29], it can be shown that for $\widehat{\mu}_{1}>\widehat{\lambda}_{1}$, the function $A\left(x, Y_{0}(x)\right) G(x)$ can be analytically continued at least up to the circle $|x|=\widehat{\mu}_{1} / \widehat{\lambda}_{1}$. Therefore we may write,

$$
\operatorname{Re}\left(i \frac{A\left(x, Y_{0}(x)\right)}{B\left(x, Y_{0}(x)\right)} G(x)\right)=0, \quad|x|=\sqrt{\frac{\widehat{\mu}_{1}}{\widehat{\lambda}_{1}}}
$$

with $Y_{0}(x) \in\left[y_{1}, y_{2}\right]$. We must ensure that the function $G(x)$ is analytic inside the circle $|x|=\widehat{\mu}_{1} / \widehat{\lambda}_{1}$, so that we have to check for poles of $G(x)$ in $1 \leq x \leq \widehat{\mu}_{1} / \widehat{\lambda}_{1}$, since it is already analytic inside the unit circle $\left(H^{(0)}(x, 0)\right.$ is analytic inside the unit circle since it is a probability generating function). From the analyticity of $A\left(x, Y_{0}(x)\right) G(x)$ it follows that the poles of $G(x)$ coincides with zeros of $A\left(x, Y_{0}(x)\right)$ for $1 \leq|x| \leq \sqrt{\widehat{\mu}_{1} / \widehat{\lambda}_{1}}$.

We proceed as in [29], section $\mathrm{V}$ and for our convenience we can rewrite $A\left(x, Y_{0}(x)\right)$ as,

$$
A\left(x, Y_{0}(x)\right)=\left(1-\frac{1}{x}\right) \frac{\widehat{\mu}_{2}-\lambda_{2}\left(\lambda+\mu_{2}\right) Y_{0}(x)-\lambda_{1} \mu_{2} x}{\widehat{\mu}_{2}-\widehat{\lambda}_{2} Y_{0}(x)}\left[d_{1}+\Psi(x)\right],
$$

where

$$
\Psi(x)=\frac{\lambda_{1}\left(\lambda+\mu_{1}\right) x+\lambda_{1} \mu_{2} Y_{0}(x)-\widehat{\mu}_{1}}{\widehat{\mu}_{2}-\lambda_{2}\left(\lambda+\mu_{2}\right) Y_{0}(x)-\lambda_{1} \mu_{2} x} .
$$

Following the lines in [29] (section $\mathrm{V}$ ) it can be proved that according to system's parameters, $A\left(x, Y_{0}(x)\right)$ has at most one zero $(\neq 1)$ in $1 \leq|x| \leq \sqrt{\widehat{\mu}_{1} / \widehat{\lambda}_{1}}$, given by $\xi_{-}$(see $\left.(3.28)\right)$.

We focus on the condition under which such a zero exists. It is easy to see using (2.10), (2.11) that $K(x, y)$ and $A(x, y)$ vanish simultaneously if and only if

$$
\begin{aligned}
(1-x)\left[\lambda_{1}\left(\lambda+\mu_{1}^{*}\right) x-\mu \mu_{1}^{*}\right]+\lambda_{2}\left(\lambda+\mu_{1}^{*}\right) x(1-y) & =0, \\
y(1-x)\left[\lambda_{1}\left(d_{1}+\mu_{2}\right) x-\mu d_{1}\right]+x(1-y)\left[\lambda_{2}\left(d_{1}+\mu_{2}\right) y-\widehat{\mu}_{2}\right] & =0 .
\end{aligned}
$$

Equation (4.7) gives for $(x, y) \notin\{(0,0),(1,1)\}$,

$$
y=\frac{x\left[\lambda_{2}\left(\lambda+\mu_{1}^{*}\right)+\mu \mu_{1}^{*}\right]-\mu \mu_{1}^{*}-\lambda_{1}\left(\lambda+\mu_{1}^{*}\right) x^{2}}{\lambda_{2}\left(\lambda+\mu_{1}^{*}\right) x} .
$$

Substituting (4.9) into (4.8) will lead to (3.27). If $1<\xi_{-} \leq \sqrt{\widehat{\mu}_{1} / \widehat{\lambda}_{1}}$ then $A\left(x, Y_{0}(x)\right)$ has a unique zero in $\left(1, \sqrt{\widehat{\mu}_{1} / \widehat{\lambda}_{1}}\right]$ provided that

$$
Y_{0}\left(\xi_{-}\right)=\frac{\xi_{-}\left[\lambda_{2}\left(\lambda+\mu_{1}^{*}\right)+\mu \mu_{1}^{*}\right]-\mu \mu_{1}^{*}-\lambda_{1}\left(\lambda+\mu_{1}^{*}\right) \xi_{-}^{2}}{\lambda_{2}\left(\lambda+\mu_{1}^{*}\right) \xi_{-}} \leq \sqrt{\widehat{\mu}_{2} / \widehat{\lambda}_{2}},
$$

since that branch $Y_{0}(x)$ is such that $\left|Y_{0}(x)\right| \leq \sqrt{\widehat{\mu}_{2} / \widehat{\lambda}_{2}}, x \in C$. On the contrary, if $Y_{0}\left(\xi_{-}\right)>$ $\sqrt{\widehat{\mu}_{2} / \widehat{\lambda}_{2}}, A\left(x, Y_{0}(x)\right)$ does not vanish in $\left(1, \sqrt{\widehat{\mu}_{1} / \widehat{\lambda}_{1}}\right]$. 
Thus, define for $k=0,1\left(k=0(1)\right.$ means that $A\left(x, Y_{0}(x)\right)$ does not vanish (vanishes respectively) in $\left.\left(1, \sqrt{\widehat{\mu}_{1} / \widehat{\lambda}_{1}}\right]\right)$.

$$
U(x):=\frac{A\left(x, Y_{0}(x)\right)}{B\left(x, Y_{0}(x)\right)\left(x-\xi_{-}\right)^{k}}, \quad \tilde{G}(x):=\left(x-\xi_{-}\right)^{k} G(x),
$$

and using (4.6) we obtain,

$$
\operatorname{Re}(i U(x) \tilde{G}(x))=0, \quad|x|=\sqrt{\widehat{\mu}_{1} / \widehat{\lambda}_{1}},
$$

which defines a homogeneous Riemann-Hilbert problem on the circle $|x|=\sqrt{\widehat{\mu}_{1} / \widehat{\lambda}_{1}}$. Let the index $\chi:=-\frac{1}{\pi}[\arg U(x)]_{|x|=\sqrt{\widehat{\mu}_{1} / \widehat{\lambda}_{1}}}$. Following the lines of Theorem 7.1 in [29], it is proved that under stability conditions given in Theorem 2 , the index $\chi=0$. Thus, the solution of the homogeneous Riemann-Hilbert problem (4.10) for $|x|<\sqrt{\widehat{\mu}_{1} / \widehat{\lambda}_{1}}$ is given by,

$$
H^{(0)}(x, 0)=D\left(x-\xi_{-}\right)^{-k} \exp \left(\frac{1}{2 i \pi} \int_{|t|=\sqrt{\widehat{\mu}_{1} / \widehat{\lambda}_{1}}} \frac{\log \{J(t)\}}{t-x} d t\right)+\frac{d_{2}\left(d_{1}-\mu_{1}\right)}{d_{1} d_{2}-\mu_{1} \mu_{2}} H^{(0)}(0,0),
$$

where $D$ is a constant and $J(z)=\frac{\overline{U(z)}}{U(z)},|z|=\sqrt{\widehat{\mu}_{1} / \widehat{\lambda}_{1}}$. Setting $x=0$ in (4.11) we obtain $D$ in term of $H^{(0)}(0,0)$. In particular,

$$
D=\frac{\mu_{1} \mu_{2}^{*}(-\xi)^{k}}{\mu_{1} \mu_{2}-d_{1} d_{2}} \exp \left(-\frac{1}{2 i \pi} \int_{|t|=\sqrt{\widehat{\mu}_{1} / \widehat{\lambda}_{1}}} \frac{\log \{J(t)\}}{t} d t\right) H^{(0)}(0,0) .
$$

Then, substituting back in (4.11), setting $x=1$, and combining with the second in (3.1), we get the constants $D$ and $H^{(0)}(0,0)$. Indeed, substituting $D$ in the second on (3.1) we obtain

$$
\begin{aligned}
H^{(0)}(0,0)= & \frac{\mu_{1}\left(\widehat{\mu}_{2}-\lambda\left(\lambda_{2}+\mu_{2}\right)\right)-d_{2}\left(\widehat{\mu}_{1}-\lambda\left(\lambda_{1}+\mu_{1}\right)\right)}{\widehat{\mu}_{1} \mu_{2}^{*}}\left(\frac{\xi_{-}-1}{\xi_{-}}\right)^{k} \\
& \times \exp \left(-\frac{1}{2 i \pi} \int_{|t|=\sqrt{\widehat{\mu}_{1} / \widehat{\lambda}_{1}}} \frac{\log \{J(t)\}}{t(t-1)} d t\right)
\end{aligned}
$$

so that

$$
\begin{aligned}
H^{(0)}(x, 0)= & \frac{\mu_{1}\left(\widehat{\mu}_{2}-\lambda\left(\lambda_{2}+\mu_{2}\right)\right)-d_{2}\left(\widehat{\mu}_{1}-\lambda\left(\lambda_{1}+\mu_{1}\right)\right)}{\widehat{\mu}_{1} \mu_{2}^{*}\left(\mu_{1} \mu_{2}-d_{1} d_{2}\right)}\left[\left(\frac{\xi_{-}-1}{\xi_{-}-x}\right)^{k} \mu_{1} \mu_{2}^{*}\right. \\
& \times \exp \left(\frac{1}{2 i \pi} \int_{|t|=\sqrt{\widehat{\mu}_{1} / \widehat{\lambda}_{1}}} \frac{\log \{J(t)\}(x-1)}{(t-1)(t-x)} d t\right) \\
& \left.-d_{2}\left(d_{1}-\mu_{1}\right)\left(\frac{\xi_{-}-1}{\xi_{-}}\right)^{k} \exp \left(\frac{-1}{2 i \pi} \int_{|t|=\sqrt{\widehat{\mu}_{1} / \widehat{\lambda}_{1}}} \frac{\log \{J(t)\}}{t(t-1)} d t\right)\right] .
\end{aligned}
$$

Using a similar approach we can also determine $H^{(0)}(0, y)$ by solving another RiemannHilbert boundary value problem on the circle $|y|=\sqrt{\widehat{\mu}_{2} / \widehat{\lambda}_{2}}$. Then, using the fundamental functional equation we obtain $H^{(0)}(x, y)$, and substituting back in $(2.6), H^{(1)}(x, y)$ is also uniquely determined. 
4.1.1. Performance metrics We proceed with the computation of some important metrics such as the expected number of customers in orbits 1 and 2, and the probability of an empty system (i.e., $P\left(N_{1}=0, N_{2}=0, C=0\right)$ ). Clearly,

$$
\begin{aligned}
E\left(N_{1}\right)= & \sum_{i=1}^{\infty} i\left[\sum_{j=0}^{\infty} p_{i, j}(0)+\sum_{j=0}^{\infty} p_{i, j}(1)\right]=\left.\frac{d}{d x} H^{(0)}(x, 1)\right|_{x=1}+\left.\frac{d}{d x} H^{(1)}(x, 1)\right|_{x=1}, \\
E\left(N_{2}\right)= & \sum_{j=1}^{\infty} j\left[\sum_{i=0}^{\infty} p_{i, j}(0)+\sum_{j=0}^{\infty} p_{i, j}(1)\right]=\left.\frac{d}{d y} H^{(0)}(1, y)\right|_{y=1}+\left.\frac{d}{d y} H^{(1)}(1, y)\right|_{y=1}, \\
P\left(N_{1}=\right. & \left.0, N_{2}=0, C=0\right)=H^{(0)}(0,0) \\
= & \frac{\mu_{1}\left(\widehat{\mu}_{2}-\lambda\left(\lambda_{2}+\mu_{2}\right)\right)-d_{2}\left(\widehat{\mu}_{1}-\lambda\left(\lambda_{1}+\mu_{1}\right)\right)}{\widehat{\mu}_{1} \mu_{2}^{*}\left(\mu_{1} \mu_{2}-d_{1} d_{2}\right)}\left(\frac{\xi_{-}-1}{\xi_{-}}\right)^{k} \\
& \times\left[\mu_{2}^{*} \mu_{1}+\mu_{1}^{*} d_{2}\right] \exp \left(\frac{-1}{2 i \pi} \int_{|t|=\sqrt{\widehat{\mu}_{1} / \widehat{\lambda}_{1}}} \frac{\log \{J(t)\}}{t(t-1)} d t\right) .
\end{aligned}
$$

Moreover, by differentiating (4.13) with respect to $x$, setting $x=1$ implies

$$
\begin{aligned}
\left.\frac{d}{d x} H^{(0)}(x, 0)\right|_{x=1}= & \frac{\mu_{1}\left(\widehat{\mu}_{2}-\lambda\left(\lambda_{2}+\mu_{2}\right)\right)-d_{2}\left(\widehat{\mu}_{1}-\lambda\left(\lambda_{1}+\mu_{1}\right)\right)}{\mu\left(\mu_{1} \mu_{2}-d_{1} d_{2}\right)} \\
& \times\left(\frac{k}{\xi_{-}-1}+\frac{1}{2 i \pi} \int_{|t|=\sqrt{\widehat{\mu}_{1} / \widehat{\lambda}_{1}}} \frac{\log \{J(t)\}}{(t-1)^{2}} d t\right) .
\end{aligned}
$$

Using (2.10) and setting $y=1$ we derive

$$
\begin{aligned}
H^{(0)}(x, 1)= & \frac{\lambda_{1}\left(d_{1}+\mu_{2}\right) x-\mu d_{1}}{\widehat{\lambda}_{1} x-\widehat{\mu}_{1}} H^{(0)}(x, 0)+\frac{\lambda_{1}\left(d_{2}+\mu_{1}\right) x-\widehat{\mu}_{1}}{\widehat{\lambda}_{1} x-\widehat{\mu}_{1}} H^{(0)}(0,1) \\
& +\frac{\mu d_{1}-\lambda_{1}\left(d_{1}+d_{2}\right) x}{\widehat{\lambda}_{1} x-\widehat{\mu}_{1}} H^{(0)}(0,0)
\end{aligned}
$$

Differentiating (4.17) with respect to $x$, setting $x=1$ yields

$$
\begin{aligned}
\left.\frac{d}{d x} H^{(0)}(x, 1)\right|_{x=1}= & \frac{\lambda_{1} \mu}{\left(\widehat{\lambda}_{1}-\widehat{\mu}_{1}\right)^{2}}\left[\left(d_{1}\left(\lambda+\mu_{2}\right)-\mu_{1} \mu_{2}\right) H^{(0)}(1,0)+\mu_{1}\left(\lambda+\mu_{2}^{*}\right) H^{(0)}(0,1)\right. \\
& \left.+\left(\mu_{1} d_{2}-d_{1}\left(\lambda+\mu_{2}\right)\right) H^{(0)}(0,0)\right]+\left.\frac{\lambda_{1}\left(d_{1}+\mu_{2}\right)-\mu d_{1}}{\widehat{\lambda}_{1}-\widehat{\mu}_{1}} \frac{d}{d x} H^{(0)}(x, 0)\right|_{x=1},
\end{aligned}
$$

where $H^{(0)}(0,1), H^{(0)}(1,0),\left.\frac{d}{d x} H^{(0)}(x, 0)\right|_{x=1}$ and $H^{(0)}(0,0)$ can be found in $(3.1),(4.16)$ and (4.12), respectively. Finally, using (2.6):

$$
\left.\frac{d}{d x} H^{(1)}(x, 1)\right|_{x=1}=\left.\frac{\alpha}{\mu} \frac{d}{d x} H^{(0)}(x, 1)\right|_{x=1}-\left.\frac{d_{1}+\mu_{2}}{\mu} \frac{d}{d x} H^{(0)}(x, 0)\right|_{x=1} .
$$


Thus, using (4.18), (4.19) we conclude that

$$
\begin{aligned}
E\left(N_{1}\right)= & \left.\frac{\mu_{2}\left(\lambda_{1}+\mu_{1}^{*}\right)-d_{1}\left(\lambda_{2}+\mu\right)}{\widehat{\lambda}_{1}-\widehat{\mu}_{1}} \frac{d}{d x} H^{(0)}(x, 0)\right|_{x=1}+H^{(0)}(1,0) \frac{\lambda_{1} \mu\left[d_{1}\left(\lambda+\mu_{2}\right)-\mu_{1} \mu_{2}\right]}{\left(\widehat{\lambda}_{1}-\widehat{\mu}_{1}\right)^{2}} \\
& +H^{(0)}(0,1) \frac{\lambda_{1} \widehat{\mu}_{1}\left(\lambda+\mu_{2}^{*}\right)}{\left(\widehat{\lambda}_{1}-\widehat{\mu}_{1}\right)^{2}}+H^{(0)}(0,0) \frac{\lambda_{1} \mu\left[\mu_{1} d_{2}-d_{1}\left(\lambda+\mu_{2}\right)\right]}{\left(\widehat{\lambda}_{1}-\widehat{\mu}_{1}\right)^{2}} .
\end{aligned}
$$
Then,

To derive $E\left(N_{2}\right)$, we need to obtain $\left.\frac{d}{d y} H^{(0)}(0, y)\right|_{y=1}$, and this can be done using (4.4).

$$
\begin{aligned}
\left.\frac{d}{d y} W(y)\right|_{y=1}= & -\left.\lim _{y \rightarrow 1} \frac{A\left(X_{0}(y), y\right)}{B\left(X_{0}(y), y\right)} \frac{d}{d y} G(x)\right|_{x=1} X_{0}^{\prime}(1)-\lim _{y \rightarrow 1} \frac{d}{d y} \frac{A\left(X_{0}(y), y\right)}{B\left(X_{0}(y), y\right)} G(1) \Rightarrow \\
\left.\frac{d}{d y} H^{(0)}(0, y)\right|_{y=1}=- & \left.\lim _{y \rightarrow 1} \frac{A\left(X_{0}(y), y\right)}{B\left(X_{0}(y), y\right)} \frac{d}{d y} H^{(0)}(x, 0)\right|_{x=1} X_{0}^{\prime}(1) \\
& -\lim _{y \rightarrow 1} \frac{d}{d y} \frac{A\left(X_{0}(y), y\right)}{B\left(X_{0}(y), y\right)}\left[H^{(0)}(1,0)-\frac{d_{2}\left(d_{1}-\mu_{1}\right)}{d_{1} d_{2}-\mu_{1} \mu_{2}} H^{(0)}(0,0)\right] \\
= & -\left.\lim _{y \rightarrow 1} \frac{A\left(X_{0}(y), y\right)}{B\left(X_{0}(y), y\right)} \frac{d}{d y} H^{(0)}(x, 0)\right|_{x=1} X_{0}^{\prime}(1) \\
& -\lim _{y \rightarrow 1} \frac{d}{d y} \frac{A\left(X_{0}(y), y\right)}{B\left(X_{0}(y), y\right)}\left[\frac{\mu_{1}\left(\widehat{\mu}_{2}-\lambda\left(\lambda_{2}+\mu_{2}\right)\right)+d_{2}\left(\lambda\left(\lambda_{1}+\mu_{1}\right)-\widehat{\mu}_{1}\right)}{\mu}\right],
\end{aligned}
$$

where using the L'Hospital rule we can obtain after some algebra

$$
\begin{aligned}
& \lim _{y \rightarrow 1} \frac{A\left(X_{0}(y), y\right)}{B\left(X_{0}(y), y\right)}=\frac{\lambda_{2}\left(d_{1}+\mu_{2}\right)-\widehat{\mu}_{2}+\left(\lambda_{1}\left(d_{1}+\mu_{2}\right)-\mu d_{1}\right) X_{0}^{\prime}(1)}{\lambda_{2}\left(d_{2}+\mu_{1}\right)-\mu d_{2}+\left(\lambda_{1}\left(d_{2}+\mu_{1}\right)-\widehat{\mu}_{1}\right) X_{0}^{\prime}(1)}, \\
& X_{0}^{\prime}(1)=\frac{\widehat{\lambda}_{2}-\widehat{\mu}_{2}}{\widehat{\mu}_{1}-\widehat{\lambda}_{1}}, \\
& X_{0}^{\prime \prime}(1)=\frac{2\left[\left(\widehat{\mu}_{1}+\widehat{\mu}_{2}-2\left(\widehat{\lambda}_{1}+\widehat{\lambda}_{2}\right)\right) \mu^{2} \mu_{1} \mu_{2}+\widehat{\lambda}_{1}^{2} \widehat{\mu}_{2}+\widehat{\lambda}_{2}^{2} \widehat{\mu}_{1}\right]}{\left(\widehat{\mu}_{1}-\widehat{\lambda}_{1}\right)^{3}},
\end{aligned}
$$

and

$$
\begin{aligned}
\lim _{y \rightarrow 1} & \frac{d}{d y} \frac{A\left(X_{0}(y), y\right)}{B\left(X_{0}(y), y\right)}=\frac{\mu}{2\left(\lambda_{2}\left(d_{2}+\mu_{1}\right)-\mu d_{2}+\left(\lambda_{1}\left(d_{2}+\mu_{1}\right)-\widehat{\mu}_{1}\right) X_{0}^{\prime}(1)\right)^{2}} \\
& \times\left\{2\left[\mu_{2}\left(d_{2}+\mu_{1}-d_{2}\left(d_{1}+\mu_{2}\right)\right)+X_{0}^{\prime}(1)\left(d_{1}\left(d_{2}+\mu_{1}\right)-\mu_{1}\left(d_{1}+\mu_{2}\right)\right)\right]\left(\lambda_{2}+\left(X_{0}^{\prime}(1)\right)^{2}\right)\right. \\
& \left.-X_{0}^{\prime \prime}(1)\left[\left(d_{1}+\mu_{2}\right)\left(\lambda_{1} d_{2}-\lambda_{2} \mu_{1}\right)+\left(d_{2}+\mu_{1}\right)\left(\lambda_{2} d_{1}-\lambda_{1} \mu_{2}\right)+\mu\left(\mu_{1} \mu_{2}-d_{1} d_{2}\right)\right]\right\} .
\end{aligned}
$$

Similarly,

$$
\begin{aligned}
\left.\frac{d}{d y} H^{(1)}(1, y)\right|_{y=1}= & \left.\frac{\alpha}{\mu} \frac{d}{d y} H^{(0)}(1, y)\right|_{y=1}-\left.\frac{d_{2}+\mu_{1}}{\mu} \frac{d}{d y} H^{(0)}(0, y)\right|_{y=1}, \\
\left.\frac{d}{d y} H^{(0)}(1, y)\right|_{y=1}= & \frac{\lambda_{2} \mu}{\left(\widehat{\lambda}_{2}-\widehat{\mu}_{2}\right)^{2}}\left[\left(d_{2}\left(\lambda+\mu_{1}\right)-\mu_{1} \mu_{2}\right) H^{(0)}(0,1)+\mu_{2}\left(\lambda+\mu_{1}^{*}\right) H^{(0)}(1,0)\right. \\
& \left.+\left(\mu_{2} d_{1}-d_{2}\left(\lambda+\mu_{1}\right)\right) H^{(0)}(0,0)\right]+\left.\frac{\lambda_{2}\left(d_{2}+\mu_{1}\right)-\mu d_{2}}{\widehat{\lambda}_{2}-\widehat{\mu}_{2}} \frac{d}{d y} H^{(0)}(0, y)\right|_{y=1} .
\end{aligned}
$$


Therefore,

$$
\begin{aligned}
E\left(N_{2}\right)= & \left.\frac{\mu_{1}\left(\lambda_{2}+\mu_{2}^{*}\right)-d_{2}\left(\lambda_{1}+\mu\right)}{\widehat{\lambda}_{2}-\widehat{\mu}_{2}} \frac{d}{d y} H^{(0)}(0, y)\right|_{y=1}+H^{(0)}(0,1) \frac{\lambda_{2} \mu\left[d_{2}\left(\lambda+\mu_{1}\right)-\mu_{1} \mu_{2}\right]}{\left(\widehat{\lambda}_{2}-\widehat{\mu}_{2}\right)^{2}} \\
& +H^{(0)}(1,0) \frac{\lambda_{2} \widehat{\mu}_{2}\left(\lambda+\mu_{1}^{*}\right)}{\left(\widehat{\lambda}_{2}-\widehat{\mu}_{2}\right)^{2}}+H^{(0)}(0,0) \frac{\lambda_{2} \mu\left[\mu_{2} d_{1}-d_{2}\left(\lambda+\mu_{1}\right)\right]}{\left(\widehat{\lambda}_{2}-\widehat{\mu}_{2}\right)^{2}}
\end{aligned}
$$

Finally, recall that (see Lemma 1) $P(C=1)=\frac{\lambda}{\mu}$.

\subsection{A Dirichlet Boundary Value Problem}

Consider now the case where $\mu_{1} \mu_{2}=d_{1} d_{2}$. Thus, there is a $\xi \in[0,1]$, such that $\mu_{1}=\xi \mu_{1}^{*}$, $\mu_{2}=(1-\xi) \mu_{2}^{*}$. Assume also that the system is in steady state (see $(3.2)$ ), that is, $\rho<1$. Then, it is easily seen that

$$
\begin{aligned}
B(x, y) & =-\frac{\xi}{1-\xi} A(x, y), \\
C(x, y) & =\mu_{2}^{*} x\left[\lambda_{1} y(1-x)+\left(\lambda_{2} y-\mu\right)(1-y)\right]-A(x, y) \\
& =\mu_{1}^{*} y\left[\lambda_{2} x(1-y)+\left(\lambda_{1} x-\mu\right)(1-x)\right]-B(x, y)
\end{aligned}
$$

For pairs $(x, y),|x| \leq 1,|y| \leq 1, K(x, y)=0$, the functional Eq. (2.9) reduces to

$$
(1-\xi) H^{(0)}(x, 0)-\xi H^{(0)}(0, y)+(1-\rho)(1-\xi) \frac{C(x, y)}{A(x, y)}=0 .
$$

Since $\operatorname{Im}\left(H^{(0)}(0, y)\right)=0$ for $y \in\left[y_{1}, y_{2}\right]$, we conclude in,

$$
\operatorname{Re}\left(i H^{(0)}(x, 0)\right)=f(x),|x|=\sqrt{\frac{\widehat{\mu}_{1}}{\widehat{\lambda}_{1}}}
$$

where

$$
\left.f(x)=(1-\rho) \operatorname{Im}\left(\frac{\mu_{2}^{*} x\left[\left(\mu-\lambda_{2} Y_{0}(x)\right)\left(1-Y_{0}(x)\right)-\lambda_{1} Y_{0}(x)(1-x)\right]}{A\left(x, Y_{0}(x)\right)}\right]\right)
$$

Clearly, if $A\left(x, Y_{0}(x)\right)$ has no zero for $|x|<\sqrt{\frac{\widehat{\mu}_{1}}{\widehat{\lambda}_{1}}}$, then $H^{(0)}(x, 0)$ is analytic in $|x|<\sqrt{\frac{\widehat{\mu}_{1}}{\widehat{\lambda}_{1}}}$ and continuous on the boundary, and (4.24) represents a Dirichlet problem on a circle. If there is a root, say $x_{0}$, of $A\left(x, Y_{0}(x)\right)$ for $|x|<\sqrt{\frac{\widehat{\mu}_{1}}{\lambda_{1}}}$, then another Dirichlet problem can be defined for the function $\left(x-x_{0}\right)^{k} H^{(0)}(x, 0), k=0,1$. Thus, we have the following problem: Determine a function $H^{(0)}(x, 0)$ such that

1. $H^{(0)}(x, 0)$ is regular for $|x|=\sqrt{\widehat{\mu}_{1} / \widehat{\lambda}_{1}}$, and continuous for $|x| \leq \sqrt{\widehat{\mu}_{1} / \widehat{\lambda}_{1}}$.

2. $R e\left(i H^{(0)}(x, 0)\right)=f(x),|x|=\sqrt{\widehat{\mu}_{1} / \widehat{\lambda}_{1}}$. 
Its solution is given by (see $[33,45])$

$$
H^{(0)}(x, 0)=-\frac{1-\rho}{2 \pi} \int_{|t|=\sqrt{\widehat{\mu}_{1} / \widehat{\lambda}_{1}}} u(t) \frac{t+x}{t-x} \frac{d t}{t}+Z,|x|<\sqrt{\widehat{\mu}_{1} / \widehat{\lambda}_{1}},
$$

where

$$
u(t)=\operatorname{Im}\left(\frac{\mu_{2}^{*} t\left[\left(\mu-\lambda_{2} Y^{*}(t)\right)(1-h(t))-\lambda_{1} Y^{*}(t)(1-t)\right]}{A\left(x, Y^{*}(t)\right)}\right),
$$

$|t|=\sqrt{\widehat{\mu}_{1} / \widehat{\lambda}_{1}}$ and $Z$ a constant to be determined. Setting $x=0$ in (4.25) we get

$$
Z=(1-\rho)\left[1+\frac{1}{2 \pi} \int_{|t|=\sqrt{\widehat{\mu}_{1} / \widehat{\lambda}_{1}}} u(t) \frac{d t}{t}\right] .
$$

Let $x=\sqrt{\widehat{\mu}_{1} / \widehat{\lambda}_{1}} e^{i \theta}$. Then, the characteristic Eq. (2.10) becomes

$$
\widehat{\lambda}_{2} y^{2}-\left(\widehat{\lambda}+\widehat{\mu}_{1}+\widehat{\mu}_{2}\right) y+\widehat{\mu}_{2}=0
$$

and its solution with the smallest modulus

$$
y=y(\theta):=Y_{0}(x)=\frac{\widehat{\lambda}_{2}+\widehat{\mu}_{2}+a-\sqrt{\left(\widehat{\lambda}_{2}+\widehat{\mu}_{2}+a\right)^{2}-4 \widehat{\lambda}_{2} \widehat{\mu}_{2}}}{2 \widehat{\lambda}_{2}},
$$

is in $\left[y_{1}, y_{2}\right]$ for $|x|=\sqrt{\widehat{\mu}_{1} / \widehat{\lambda}_{1}}$, and $a=\widehat{\lambda}_{1}+\widehat{\mu}_{1}-2 \sqrt{\widehat{\lambda}_{1} \widehat{\mu}_{1}} \cos \theta$. Then,

$$
H^{(0)}(x, 0)=-\frac{1-\rho}{2 \pi} \int_{-\pi}^{\pi} \widehat{T}(\theta) \frac{\sqrt{\widehat{\mu}_{1} / \widehat{\lambda}_{1}} e^{i \theta}+x}{\sqrt{\widehat{\mu}_{1} / \widehat{\lambda}_{1}} e^{i \theta}-x} d \theta+Z, \quad|x|<\sqrt{\widehat{\mu}_{1} / \widehat{\lambda}_{1}},
$$

where $\widehat{T}(\theta)=u\left(\sqrt{\widehat{\mu}_{1} / \widehat{\lambda}_{1}} e^{i \theta}\right)$. Using the facts that $y(\theta)$ is an even function of $\theta$ and $T(\theta)$ is an odd function of $\theta$, we obtain for $|x|<\sqrt{\widehat{\mu}_{1} / \widehat{\lambda}_{1}}$

$$
H^{(0)}(x, 0)=\frac{2 \sqrt{\widehat{\mu}_{1} \widehat{\lambda}_{1}}(1-\rho)}{\pi} \int_{0}^{\pi} \frac{\widehat{T}(\theta) x \sin \theta}{\widehat{\mu}_{1}-2 \sqrt{\widehat{\mu}_{1} \widehat{\lambda}_{1}} \cos \theta+\widehat{\lambda}_{1} x^{2}} d \theta+Z .
$$

Similarly, we can also obtain the $H^{(0)}(0, y),|y|<\sqrt{\widehat{\mu}_{2} / \widehat{\lambda}_{2}}$, by solving another Dirichlet problem. Substituting $H^{(0)}(0, y), H^{(0)}(x, 0)$, into $(2.6)$, $(2.7)$, we can determine $H^{(1)}(x, y)$, $H^{(0)}(x, y)$.

\section{ASYMPTOTIC ANALYSIS}

In the following, we focus on the model analyzed in Section 4 for $\mu_{1} \mu_{2}=d_{1} d_{2}$, that is, $\mu_{1}=\xi \mu_{1}^{*}, \mu_{2}=(1-\xi) \mu_{2}^{*}$. Similar results can be deduced for the general case.

In $[42,43]$, the kernel method was applied to study the exact tail asymptotic properties for random walks in the quarter plane. Song, Liu, and Zhao [52] performed asymptotic 
analysis for the model in [7]. In this section, we fill the gap and provide asymptotic results for a retrial model with two input streams and coupled orbit queues, based on the random walk approach presented in Section 3.1. Using the fundamental Eq. (3.17), we can obtain asymptotic properties at the dominant singularity for the generating functions $\pi_{1}^{(1)}(x)$, $\pi_{2}^{(1)}(y)$, with the aid of which, we can identify the exact tail properties for $p_{i, j}(1)$, through the Tauberian-like theorem. Then, using (2.6) we can also characterize the exact tail asymptotic properties for $p_{i, j}(0)$.

We proceed with the investigation of the dominant singularity of the $\pi_{1}^{(1)}(x)$ (similar results can be obtained for $\left.\pi_{2}^{(1)}(y)\right)$. The dominant singularity of $\pi_{1}^{(1)}(x)$ is either a branch point of the kernel equation $k^{(1)}(x, y)=0$ or a pole of the function $\pi_{1}^{(1)}(x)[42,43]$. Clearly, $k^{(1)}(x, y)=-\alpha K(x, y)$, and thus, the branch points of $k^{(1)}(x, y)=0$ coincides with the branch points of $K(x, y)=0$, thoroughly investigated in Section 3.2 (see also [52] pp. 10-11).

Since the censored MC on the states of a busy server is a standard random walk in the quarter plane, we can employ literature results on the analysis of the dominant singularity of $\pi_{1}^{(1)}(x)$. Except the branch point

$$
x_{3}=\frac{\widehat{\lambda}_{1}+\xi \mu \mu_{1}^{*}+\left(\sqrt{\widehat{\lambda}_{2}}-\sqrt{(1-\xi) \mu \mu_{2}^{*}}\right)^{2}-\sqrt{V}}{2 \widehat{\lambda}_{1}},
$$

where

$$
V=\left[\left(\widehat{\lambda}_{1}+\xi \mu \mu_{1}^{*}\right)+\left(\sqrt{\widehat{\lambda}_{2}}-\sqrt{(1-\xi) \mu \mu_{2}^{*}}\right)^{2}\right]^{2}-4 \widehat{\lambda}_{1} \xi \mu_{1}^{*},
$$

the other candidate for the dominant singularity is a pole of $\pi_{1}^{(1)}(x)$ in the interval $\left(1, x_{3}\right]$, which can only be the zero of $A\left(x, Y_{0}(X)\right)$.

Note that for this censored random walk, $p_{i, j}, p_{i, j}^{(1)}$ are not $X$-shaped, and according to [43], Theorem 4.5, either $A\left(x, Y_{0}(x)\right)$ has no zeros with modulus in $\left(1, x_{3}\right]$, or it has only one, say $x^{*}$ in $\left(1, x_{3}\right]$. In the former case, let $x^{*}=+\infty$ (in this case, obviously $x^{*}$ can never be the dominant singularity since $\left.x_{3}<+\infty\right)$. To check the existence of such a zero, instead of directly considering the equation $A\left(x, Y_{0}(x)\right)=0$, we consider the product of two functions $A\left(x, Y_{0}(x)\right)$ and $A\left(x, Y_{1}(x)\right)$, which is a polynomial:

$$
A\left(x, Y_{0}(x)\right) A\left(x, Y_{1}(x)\right)=\frac{\mu(1-\xi)(x-1)}{\alpha\left(\lambda+\mu_{1}^{*}\right)^{2}} g(x),
$$

where

$$
g(x)=\lambda \lambda_{1}\left(\lambda+\mu_{1}^{*}\right)\left(\mu_{2}^{*}-\mu_{1}^{*}\right) x^{2}+\lambda \mu_{1}^{*} x\left[\left(\lambda+\mu_{1}^{*}\right)\left(\lambda+\mu_{2}^{*}\right)+\mu\left(\mu_{1}^{*}-\mu_{2}^{*}\right)\right]-\mu\left(\mu_{1}^{*}\right)^{2}\left(\lambda+\mu_{2}^{*}\right) .
$$

It is easily checked that $g(0)<0$, and $g(1)=\mu \mu_{1}^{*} \mu_{2}^{*}\left(\lambda+\mu_{2}^{*}\right)(\rho-1)<0$ due to the stability condition (3.14). The polynomial $g(x)$ has two real roots since

$$
\begin{aligned}
\Delta_{0} & =\left(\lambda \mu_{1}^{*}\left(\lambda+\mu_{2}^{*}\right)\left(\lambda+\mu_{1}^{*}\right)\right)^{2}+\left(\lambda \mu \mu_{1}^{*}\left(\mu_{1}^{*}-\mu_{2}^{*}\right)\right)^{2}+2 \lambda\left(\lambda_{2}-\lambda_{1}\right)\left(\mu_{1}^{*}\right)^{2}\left(\lambda+\mu_{2}\right)\left(\mu_{1}^{*}-\mu_{2}^{*}\right) \\
& \geq\left(\lambda \mu_{1}^{*}\left(\lambda+\mu_{2}\right)\left(\lambda+\mu_{1}^{*}\right)-\lambda \mu \mu_{1}^{*}\left|\mu_{1}^{*}-\mu_{2}^{*}\right|\right)^{2} \geq 0,
\end{aligned}
$$

given by

$$
x_{ \pm}=\frac{-\lambda \mu_{1}^{*}\left[\mu\left(\mu_{1}^{*}-\mu_{2}^{*}\right)+\left(\lambda+\mu_{2}^{*}\right)\left(\lambda+\mu_{1}^{*}\right)\right] x \pm \sqrt{\Delta_{0}}}{2 \lambda \lambda_{1}\left(\mu_{1}^{*}-\mu_{2}^{*}\right)\left(\lambda+\mu_{1}^{*}\right)} .
$$

Moreover, these roots cannot belong to $\left(x_{2}, 1\right]$ since their product has modulus greater than 1. If $\mu_{2}^{*}-\mu_{1}^{*}>0, x_{ \pm}$are of opposite sign with $x_{+}>1$. If $\mu_{2}^{*}-\mu_{1}^{*}<0$, then they are 
both positive and $x_{-} \leq x_{3}<x_{4} \leq x_{+}$. In the former case we focus on $x_{+}$, while in the latter one in $x_{-}$.

We proceed with the case $\mu_{2}^{*}-\mu_{1}^{*}>0$; the procedure with the other case is repetitive and it is omitted. Solving $A\left(x_{+}, Y\left(x_{+}\right)\right)=0$, we get

$$
y=Y\left(x_{+}\right)=1-\frac{\left[\lambda_{1}\left(\lambda+\mu_{1}^{*}\right)+\mu \mu_{1}^{*}\right]\left(x_{+}-1\right)}{\lambda_{2}\left(\lambda+\mu_{1}^{*}\right) x_{+}} .
$$

Due to stability condition (3.14), $\frac{\lambda\left(\lambda_{1}+\mu_{1}^{*}\right)}{\mu \mu_{1}^{*}}<1$. Thus, $\lambda_{1}\left(\lambda+\mu_{1}^{*}\right)<\mu \mu_{1}^{*}$, and

$$
g\left(\frac{\mu \mu_{1}^{*}}{\lambda_{1}\left(\lambda+\mu_{1}^{*}\right)}\right)=\frac{\lambda \lambda_{1} \mu\left(\mu_{1}^{*}\right)^{2}\left(\lambda+\mu_{2}^{*}\right)}{\lambda_{1}}>0 .
$$

Hence, $1<x_{+}<\frac{\mu \mu_{1}^{*}}{\lambda_{1}\left(\lambda+\mu_{1}^{*}\right)}$, which means $Y\left(x_{+}\right)>1$.

The following results are simple generalizations of the results found in [42] (Lemmas $2.3,4.4$, pp. 12-13) and will be used in the following.

\section{LEMMA 3:}

1. If $\widehat{\mu}_{2}>\widehat{\lambda}_{2}$, the value of $x^{*}$ depends on the value of $x_{+}$:

(a) For $x_{+} \in\left(1, \widehat{\mu}_{1} / \widehat{\lambda}_{1}\right], x^{*}=\infty$;

(b) For $x_{+} \in\left(\widehat{\mu}_{1} / \widehat{\lambda}_{1}, \min \left(x_{3}, \frac{\mu \mu_{1}^{*}}{\lambda_{1}\left(\lambda+\mu_{1}^{*}\right)}\right)\right), x^{*}=x_{+}<x_{3}$ if $Y\left(x_{+}\right)<\sqrt{\widehat{\mu}_{2} / \widehat{\lambda}_{2}}$, and $x^{*}=\infty$ otherwise;

(c) For $x_{+}=x_{3}<\frac{\mu \mu_{1}^{*}}{\lambda_{1}\left(\lambda+\mu_{1}^{*}\right)}, Y\left(x_{+}\right)=\sqrt{\widehat{\mu}_{2} / \widehat{\lambda}_{2}}$ and $x^{*}=x_{3}=x_{+}$;

(d) For $x_{3}<x_{+}<\frac{\mu \mu_{1}^{*}}{\lambda_{1}\left(\lambda+\mu_{1}^{*}\right)}, x^{*}=\infty$.

2. If $\widehat{\mu}_{2} \leq \widehat{\lambda}_{2}, x^{*}=\infty$.

Proof: The proof is completely based on [42] (Lemma 4.3).

\section{LEMMA 4:}

1. If $\widehat{\mu}_{2}>\widehat{\lambda}_{2}, x_{+} \in\left(\widehat{\mu}_{1} / \widehat{\lambda}_{1}, \min \left(x_{3}, \frac{\mu \mu_{1}^{*}}{\lambda_{1}\left(\lambda+\mu_{1}^{*}\right)}\right)\right)$ and $Y\left(x_{+}\right)<\sqrt{\widehat{\mu}_{2} / \widehat{\lambda}_{2}}$, the dominant singularity $x_{d o m}=x^{*}=x_{+}<x_{3}$, which is a pole.

2. If $\widehat{\mu}_{2}>\widehat{\lambda}_{2}$ and $x_{+}=x_{3} \in\left(\frac{\widehat{\mu}_{1}}{\widehat{\lambda}_{1}}, \frac{\mu \mu_{1}^{*}}{\lambda_{1}\left(\lambda+\mu_{1}^{*}\right)}\right)$, the dominant singularity $x_{\text {dom }}=x^{*}=$ $x_{+}=x_{3}$, which is both a pole and a branch point.

3. Under one of the following three (a) $\widehat{\mu}_{2} \leq \widehat{\lambda}_{2}$; (b) $\widehat{\mu}_{2}>\widehat{\lambda}_{2}$ and $x_{+} \in\left(1, \widehat{\mu}_{1} / \widehat{\lambda}_{1}\right]$; and (c) $\widehat{\mu}_{2}>\widehat{\lambda}_{2}, x_{+} \in\left(\widehat{\mu}_{1} / \widehat{\lambda}_{1}, \min \left(x_{3}, \frac{\mu \mu_{1}^{*}}{\lambda_{1}\left(\lambda+\mu_{1}^{*}\right)}\right)\right)$ and $Y\left(x_{+}\right) \geq \sqrt{\widehat{\mu}_{2} / \widehat{\lambda}_{2}}$, the dominant singularity $x_{\text {dom }}=x_{3}<x^{*}=\infty$, which is a branch point.

The following theorem proves that there are three types of detailed asymptotic properties of $\pi_{1}^{(1)}(x)$ as $x$ approaches the dominant singularity. 
THEOREM 3: The behavior of $\pi_{1}^{(1)}(x)$ at the dominant singularity is given as

1. If $x_{d o m}=x^{*}=x_{+}<x_{3}$, then

$$
\lim _{x \rightarrow x_{+}}\left(1-\frac{x}{x_{+}}\right) \pi_{1}^{(1)}(x)=C_{1,0}
$$

where

$$
\begin{aligned}
C_{1,0}= & \frac{\alpha\left(\lambda+\mu_{1}^{*}\right) a^{(1)}\left(x_{+}, Y_{0}\left(x_{+}\right)\right)}{\mu(1-\xi) \lambda \lambda_{1}\left(\mu_{2}^{*}-\mu_{1}^{*}\right) x_{+}\left(x_{+}-1\right)\left(x_{+}-x_{-}\right)} \\
& \times\left[b^{(1)}\left(x_{+}, Y_{0}\left(x_{+}\right)\right) \pi_{2}^{(1)}\left(Y_{0}\left(x_{+}\right)\right)+c^{(1)}\left(x_{+}, Y_{0}\left(x_{+}\right)\right) \pi^{(1)}(0,0)\right] .
\end{aligned}
$$

2. If $x_{d o m}=x^{*}=x_{+}=x_{3}$, then

$$
\lim _{x \rightarrow x_{\text {dom }}} \sqrt{1-\frac{x}{x_{d o m}}} \pi_{1}^{(1)}(x)=C_{2,0}
$$

where

$$
C_{2,0}=\frac{2}{\lambda_{1}} \frac{b^{(1)}\left(x_{d o m}, Y_{0}\left(x_{d o m}\right)\right) \pi_{2}^{(1)}\left(Y_{0}\left(x_{d o m}\right)\right)+c^{(1)}\left(x_{d o m}, Y_{0}\left(x_{d o m}\right)\right) \pi^{(1)}(0,0)}{\sqrt{x_{d o m}\left(x_{d o m}-x_{1}\right)\left(x_{d o m}-x_{2}\right)\left(x_{4}-x_{d o m}\right)}} .
$$

3. If $x_{d o m}=x_{3}<x^{*}=+\infty$, then

$$
\lim _{x \rightarrow x_{3}} \sqrt{1-\frac{x}{x_{3}}} \frac{d}{d x} \pi_{1}^{(1)}(x)=C_{3,0},
$$

where

$$
\begin{aligned}
C_{3,0} & =-\left.\frac{q\left(x_{3}\right)}{2 x_{3}} \frac{d}{d y}\left[\frac{b^{(1)}\left(x_{3}, y\right) \pi_{2}^{(1)}(y)+c^{(1)}\left(x_{3}, y\right) \pi^{(1)}(0,0)}{a^{(1)}\left(x_{3}, y\right)}\right]\right|_{y=Y_{0}\left(x_{3}\right)}, \\
q\left(x_{3}\right) & =-\frac{\lambda_{1}}{2 \lambda_{2} x_{3}} \sqrt{x_{3}\left(x_{3}-x_{1}\right)\left(x_{3}-x_{2}\right)\left(x_{4}-x_{3}\right)} .
\end{aligned}
$$

Proof: The proof is based on [43] p. 15 and due to space economy the details are omitted.

We are going to apply the following Tauberian-like theorem in order to obtain the exact tail asymptotic properties in stationary probabilities from the corresponding asymptotic properties of the generating function. Note that using the Tauberian-like theorem, it is not necessary to fully determine the unknown generating function with the aid of the boundary value theory. For alternative approaches see [35,36]. Just for sake of clarity we state the following Tauberian-like theorem for single singularity that we are going to use (for further reading see Flajolet and Sedgewick [32]).

TheOREM 4 [32]: Let $A(z)=\sum_{n \geq 0} a_{n} z^{n}$ be analytic at zero with $R$ the radius of convergence. Suppose that $R$ is a singularity of $A(z)$ that can be continued to a $\Delta$-domain at $R$. 
If for a real number $\beta \notin\{0,1,2, \ldots\}$,

$$
\lim _{z \rightarrow R}(1-z / R)^{\beta} A(z)=g,
$$

where $g$ is a non-zero constant. Then,

$$
a_{n} \sim \frac{g}{\Gamma(\beta)} n^{\beta-1} R^{-n}
$$

where $\Gamma(\beta)$ is the value of Gamma function at $\beta$, and $a_{n} \sim$ bn is equivalent to lim $_{n}$ $\left(a_{n} / b_{n}\right)=1$.

\subsection{Exact Tail Asymptotics for a Busy Server}

The following theorem provides asymptotic properties of the boundary probabilities $p_{i, 0}(1)$, and it is a direct consequence of Theorems 3 and 4 .

THEOREM 5: If $\rho<1$ then, when $i$ is large, we have three types of tail asymptotic properties for the boundary probabilities $p_{i, 0}(1)$ :

1. Exact geometric decay: If $x_{d o m}=x^{*}=x_{+}<x_{3}$, then

$$
p_{i, 0}(1) \sim C_{1,0}\left(\frac{1}{x_{+}}\right)^{i-1}, \quad i \geq 1 ;
$$

2. Geometric decay with prefactor $i^{-1 / 2}$ : If $x_{d o m}=x^{*}=x_{+}=x_{3}$, then

$$
p_{i, 0}(1) \sim \frac{C_{2,0}}{\sqrt{\pi}} i^{-1 / 2}\left(\frac{1}{x_{d o m}}\right)^{i-1}, \quad i \geq 1 ;
$$

3. Geometric decay with prefactor $i^{-3 / 2}$ : If $x_{d o m}=x_{3}<x^{*}=+\infty$, then

$$
p_{i, 0}(1) \sim \frac{C_{3,0}}{\sqrt{\pi}} i^{-3 / 2}\left(\frac{1}{x_{3}}\right)^{i-2}, \quad i \geq 1,
$$

where $C_{i, 0}, i=1,2,3$ are given in Theorem 3 .

We proceed with the characterization of the asymptotic behavior of the marginal probability $\pi_{i}^{(1)}=\sum_{j \geq 1} p_{i, j}(1)$. We compute

$$
\begin{aligned}
\pi^{(1)}(x, 1) & =\frac{a^{(1)}(x, 1) \pi_{1}^{(1)}(x)+b^{(1)}(x, 1) \pi_{2}^{(1)}(1)+c^{(1)}(x, 1) \pi^{(1)}(0,0)}{-k^{(1)}(x, 1)} \\
& =\frac{\alpha}{\lambda+\mu_{1}^{*}} \frac{\left[\lambda_{1}\left(\lambda+\mu_{1}^{*}\right) x-\mu \mu_{1}^{*}\right] \pi_{1}^{(1)}(x)+\lambda_{1}\left(\lambda+\mu_{1}^{*}\right)\left(\pi_{2}^{(1)}(1)+\pi^{(1)}(0,0)\right)}{\xi \mu \mu_{1}^{*}\left(1-\frac{\widehat{\lambda}_{1}}{\xi \mu \mu_{1}^{*}}\right)} .
\end{aligned}
$$

Following the discussion in [52], p. 17, it can be seen that the Tauberian-like theorem can be applied for the marginal probability $\pi_{i}^{(1)}$ : 
THEOREM 6:

$$
\lim _{x \rightarrow \xi \mu \mu_{1}^{*} / \widehat{\lambda}_{1}}\left(1-\frac{x}{\xi \mu \mu_{1}^{*} / \widehat{\lambda}_{1}}\right) \pi^{(1)}(x, 1)=C_{i}
$$

where

$$
C_{i}=-\frac{(1-\xi) \mu_{2}^{*}}{\lambda+\mu_{1}^{*}} \pi_{1}^{(1)}\left(\xi \mu \mu_{1}^{*} / \widehat{\lambda}_{1}\right)+\frac{\widehat{\lambda}_{1}}{\xi \mu \mu_{1}^{*}}\left(\pi_{2}^{(1)}(1)+\pi^{(1)}(0,0)\right)
$$

The marginal probabilities $\pi_{i}^{(1)}$ has an exact geometric decay with decay rate equal to $x_{\text {dom }}=$ $\xi \mu \mu_{1}^{*} / \widehat{\lambda}_{1}$ :

$$
\pi_{i}^{(1)} \sim C_{i}\left(\frac{\widehat{\lambda}_{1}}{\xi \mu \mu_{1}^{*}}\right)^{i-1}
$$

Next, we focus on the exact tail asymptotic behavior for joint probabilities $p_{i, j}(1)$. Following the lines in Theorem 7.1 and Lemma 7.2 in [43], we conclude to the following theorem.

Theorem 7: Corresponding to the three types in Theorem 3, when $i$ is large, we have the following tail asymptotic properties for the joint probabilities $p_{i, j}(1)$ for a fixed $j$ :

1. Exact geometric decay: If $x_{d o m}=x^{*}=x_{+}<x_{3}$, then

$$
p_{i, j}(1) \sim-\frac{b_{1}\left(x_{+}\right) C_{1,0}}{c\left(x_{+}\right)}\left(\frac{1}{Y_{1}\left(x_{+}\right)}\right)^{j-1}\left(\frac{1}{x_{+}}\right)^{i-1}, j \geq 1
$$

2. Geometric decay with prefactor $i^{-1 / 2}$ : If $x_{d o m}=x^{*}=x_{+}=x_{3}$, then

$$
p_{i, j}(1) \sim-\frac{b_{1}\left(x_{d o m}\right) C_{2,0}}{\sqrt{\pi} c\left(x_{d o m}\right)}\left(\frac{1}{Y_{1}\left(x_{d o m}\right)}\right)^{j-1} i^{-1 / 2}\left(\frac{1}{x_{d o m}}\right)^{i-1}, \quad j \geq 1
$$

3. Geometric decay with prefactor $i^{-3 / 2}$ : If $x_{d o m}=x_{3}<x^{*}=+\infty$, then

$$
\begin{aligned}
& \qquad p_{i, j}(1) \sim-\frac{b_{1}\left(x_{3}\right) C_{3,0}+(j-1) l\left(x_{3}\right)}{\sqrt{\pi} c\left(x_{3}\right)}\left(\frac{1}{Y_{1}\left(x_{3}\right)}\right)^{j-1} i^{-3 / 2}\left(\frac{1}{x_{3}}\right)^{i-2}, \quad j \geq 1, \\
& \text { where } l(x)=\frac{2 \widehat{\lambda}_{2} x b_{1}(x)-\left[\widehat{\lambda}_{1} x^{2}-\left(\widehat{\lambda}+\widehat{\mu}_{1}+\widehat{\mu}_{2}\right) x+\widehat{\mu}_{1}\right]}{2 \widehat{\lambda}_{2} x c(x)} C_{3,0} \text {, and } b_{1}(x), c(x) \text { are given in }(3.18) .
\end{aligned}
$$

\subsection{Exact Tail Asymptotics for an Idle Server}

Our knowledge on the exact tail asymptotic properties of the boundary, marginal and joint distributions for a busy server, will help us to obtain the corresponding properties for an 
idle server. Clearly, setting $y=0$ in (2.6) we conclude in

$$
H^{(0)}(x, 0)=\frac{\mu}{\lambda+\mu_{1}^{*}} H^{(1)}(x, 0)+\frac{(1-\rho) \mu_{1}^{*}}{\lambda+\mu_{1}^{*}},
$$

which means that $H^{(0)}(x, 0)$ and $H^{(1)}(x, 0)$ have the same asymptotic property. Setting $y=0$ in $(2.6)$ we conclude in

$$
\begin{aligned}
\alpha H^{(0)}(x, 1)= & \mu H^{(1)}(x, 1)-\left(\mu_{1}^{*}-\mu_{2}^{*}\right)\left[(1-\xi) H^{(0)}(x, 0)-\xi H^{(0)}(0,1)\right] \\
& +(1-\rho)\left[(1-\xi) \mu_{1}^{*}+\xi \mu_{2}^{*}\right] .
\end{aligned}
$$

Substituting (5.2) into (5.3) yields

$$
\begin{aligned}
\alpha H^{(0)}(x, 1)= & \mu H^{(1)}(x, 1)-\left(\mu_{1}^{*}-\mu_{2}^{*}\right)\left[\frac{(1-\xi) \mu}{\lambda+\mu_{1}^{*}} H^{(1)}(x, 0)\right. \\
& \left.-\xi H^{(0)}(0,1)\right]+(1-\rho)\left[\frac{\mu_{1}^{*}}{\lambda+\mu_{1}^{*}}+(1-\xi) \mu_{1}^{*}+\xi \mu_{2}^{*}\right] .
\end{aligned}
$$

Thus, $H^{(0)}(x, 1)$ and $H^{(1)}(x, 1)$ have the same asymptotic property. Based on the above, we have the following conclusion:

TheOREM 8: If $\rho<1$ then,

1. For large $i$, we have three types of tail asymptotic properties for the boundary probabilities $p_{i, 0}(0)$ correspondingly.

(a) Exact geometric decay: If $x_{d o m}=x^{*}=x_{+}<x_{3}$, then

$$
p_{i, 0}(0) \sim \frac{\mu}{\lambda+\mu_{1}^{*}} C_{1,0}\left(\frac{1}{x_{+}}\right)^{i-1}, \quad i \geq 1 ;
$$

(b) Geometric decay with prefactor $i^{1 / 2}$ : If $x_{d o m}=x^{*}=x_{+}=x_{3}$, then

$$
p_{i, 0}(0) \sim \frac{\mu}{\lambda+\mu_{1}^{*}} \frac{C_{2,0}}{\sqrt{\pi}} i^{-1 / 2}\left(\frac{1}{x_{d o m}}\right)^{i-1}, \quad i \geq 1 ;
$$

(c) Geometric decay with prefactor $i^{3 / 2}$ : If $x_{d o m}=x_{3}<x^{*}=+\infty$, then

$$
p_{i, 0}(0) \sim \frac{\mu}{\lambda+\mu_{1}^{*}} \frac{C_{3,0}}{\sqrt{\pi}} i^{-3 / 2}\left(\frac{1}{x_{3}}\right)^{i-2}, \quad i \geq 1,
$$

where $C_{i, 0}$ are given in Theorem 3.

2. The tail asymptotic property of the marginal distribution $\pi_{i}^{(0)}=\sum_{j \geq 1} p_{i, j}(0)$ is determined by

$$
\pi_{i}^{(0)} \sim \frac{\mu}{\alpha} C_{i}\left(\frac{\widehat{\lambda}_{1}}{\xi \mu \mu_{1}^{*}}\right)^{i-1},
$$

where $C_{i}$ is given in Theorem 6 .

Finally, referring to the second equation in (2.4) we conclude in the following theorem for the tail asymptotic behavior of the joint probabilities $p_{i, j}(0)$ : 
TheOREM 9: Corresponding to the three types in Theorem 3, when $i$ is large, we have the following tail asymptotic properties for the joint probabilities $p_{i, j}(1)$ for a fixed $j$ :

1. Exact geometric decay: If $x_{d o m}=x^{*}=x_{+}<x_{3}$, then

$$
p_{i, j}(0) \sim-\frac{\mu}{\alpha} \frac{b_{1}\left(x_{+}\right) C_{1,0}}{c\left(x_{+}\right)}\left(\frac{1}{Y_{1}\left(x_{+}\right)}\right)^{j-1}\left(\frac{1}{x_{+}}\right)^{i-1}, \quad j \geq 1
$$

2. Geometric decay with prefactor $i^{-1 / 2}$ : If $x_{d o m}=x^{*}=x_{+}=x_{3}$, then

$$
p_{i, j}(0) \sim-\frac{\mu}{\alpha} \frac{b_{1}\left(x_{d o m}\right) C_{2,0}}{\sqrt{\pi} c\left(x_{d o m}\right)}\left(\frac{1}{Y_{1}\left(x_{d o m}\right)}\right)^{j-1} i^{-1 / 2}\left(\frac{1}{x_{d o m}}\right)^{i-1}, \quad j \geq 1 ;
$$

3. Geometric decay with prefactor $i^{-3 / 2}$ : If $x_{d o m}=x_{3}<x^{*}=+\infty$, then

$$
p_{i, j}(0) \sim-\frac{\mu}{\alpha} \frac{b_{1}\left(x_{3}\right) C_{3,0}+(j-1) l\left(x_{3}\right)}{\sqrt{\pi} c\left(x_{3}\right)}\left(\frac{1}{Y_{1}\left(x_{3}\right)}\right)^{j-1} i^{-3 / 2}\left(\frac{1}{x_{3}}\right)^{i-2}, \quad j \geq 1,
$$

where

$$
(x)=\frac{2 \widehat{\lambda}_{2} x b_{1}(x)-\left[\widehat{\lambda}_{1} x^{2}-\left(\widehat{\lambda}+\widehat{\mu}_{1}+\widehat{\mu}_{2}\right) x+\widehat{\mu}_{1}\right]}{2 \widehat{\lambda}_{2} x c(x)} C_{3,0},
$$

and $b_{1}(x), c(x)$ are given in $(3.18)$.

\section{THE MODEL WITH GENERAL SERVICE TIMES}

In the following, we consider a generalization of the main model by assuming that the service time distribution is not exponentially distributed, but instead, it is arbitrarily distributed with cdf (cumulative distribution function) $B(x)$, pdf (probability density function) $b(x)$ and Laplace-Stieltjes Transform (LST) $\beta(s)$ and mean $\bar{b}$. The assumption of arbitrary distributed service times will change our analysis, but hopefully, we are able to use the approach developed in Cohen and Boxma [17] and transform the inherent functional equation into a boundary value problem of the Riemann type.

Let $X_{i}(n)$, to be the number of type $i, i=1,2$, retrial customers in orbit $i$, just after the end of the $n$th service completion. Define by $A_{i}(n), i=1,2$, to be the number of type $i$ customers that arrive during the $n$th service service time. Denote for $\left|z_{1}\right| \leq 1,\left|z_{2}\right| \leq 1$,

$$
\pi_{m, l}=\lim _{n \rightarrow \infty} \operatorname{Pr}\left(\left(X_{1}(n), X_{2}(n)\right)=(m, l)\right), \Pi\left(z_{1}, z_{2}\right)=\sum_{m=0}^{\infty} \sum_{l=0}^{\infty} \pi_{m, l} z_{1}^{m} z_{2}^{l} .
$$

Clearly, if $r_{i}=\lambda_{i} / \lambda, i=1,2, \lambda=\lambda_{1}+\lambda_{2}$,

$$
\begin{aligned}
P\left(A_{1}=k, A_{2}=m\right) & =d_{k, m}=\int_{0}^{\infty} e^{-\lambda_{1} t} \frac{\left(\lambda_{1} t\right)^{k}}{k !} e^{-\lambda_{2} t} \frac{\left(\lambda_{2} t\right)^{k}}{m !} d B(x) \\
d^{*}\left(z_{1}, z_{2}\right) & =\sum_{m=0}^{\infty} \sum_{l=0}^{\infty} d_{k m} z_{1}^{k} z_{2}^{m}=\beta^{*}\left(\lambda\left(1-r_{1} z_{1}-r_{2} z_{2}\right)\right)
\end{aligned}
$$


Set $y:=\lambda\left(1-r_{1} z_{1}-r_{2} z_{2}\right)$. Considering the transition probabilities during successful service completion epochs we obtain,

$$
\begin{aligned}
\pi_{m, l}= & \frac{\mu_{1}}{\lambda+\mu_{1}+\mu_{2}} \sum_{k_{1}=1}^{m+1} \sum_{k_{2}=1}^{l} \pi_{k_{1}, k_{2}} d_{m+1-k_{1}, l-k_{2}}+\frac{\mu_{2}}{\lambda+\mu_{1}+\mu_{2}} \sum_{k_{1}=1}^{m} \sum_{k_{2}=1}^{l+1} \pi_{k_{1}, k_{2}} d_{m-k_{1}, l+1-k_{2}} \\
& +\frac{\lambda}{\lambda+\mu_{1}+\mu_{2}} \sum_{k_{1}=1}^{m} \sum_{k_{2}=1}^{l} \pi_{k_{1}, k_{2}} d_{m-k_{1}, l-k_{2}}+\frac{\mu_{1}^{*}}{\lambda+\mu_{1}^{*}} \sum_{k_{1}=1}^{m+1} \pi_{k_{1}, 0} d_{m+1-k_{1}, l}+\pi_{0,0} d_{m, l} \\
& +\frac{\lambda}{\lambda+\mu_{1}^{*}} \sum_{k_{1}=1}^{m} \pi_{k_{1}, 0} d_{m-k_{1}, l}+\frac{\mu_{2}^{*}}{\lambda+\mu_{2}^{*}} \sum_{k_{2}=1}^{l+1} \pi_{0, k_{2}} d_{m, l+1-k_{2}}+\frac{\lambda}{\lambda+\mu_{2}^{*}} \sum_{k_{2}=1}^{l} \pi_{0, k_{2}} d_{m, l-k_{2}} .
\end{aligned}
$$

Forming the generating functions we conclude that

$$
\widehat{K}\left(z_{1}, z_{2}\right) \Pi\left(z_{1}, z_{2}\right)=\widehat{A}\left(z_{1}, z_{2}\right) \Pi\left(z_{1}, 0\right)+\widehat{B}\left(z_{1}, z_{2}\right) \Pi\left(0, z_{2}\right)+\widehat{C}\left(z_{1}, z_{2}\right) \Pi(0,0),
$$

where

$$
\begin{aligned}
\widehat{K}\left(z_{1}, z_{2}\right) & =z_{1} z_{2}-\widetilde{K}\left(z_{1}, z_{2}\right) \\
\widehat{A}\left(z_{1}, z_{2}\right) & =z_{2} \widetilde{A}\left(z_{1}, z_{2}\right)-\widetilde{K}\left(z_{1}, z_{2}\right), \\
\widehat{B}\left(z_{1}, z_{2}\right) & =z_{1} \widetilde{B}\left(z_{1}, z_{2}\right)-\widetilde{K}\left(z_{1}, z_{2}\right), \\
\widehat{C}\left(z_{1}, z_{2}\right) & =\widetilde{K}\left(z_{1}, z_{2}\right)+z_{2}\left(r_{1} z_{1} \beta^{*}(y)-\widetilde{A}\left(z_{1}, z_{2}\right)\right)+z_{1}\left(r_{2} z_{2} \beta^{*}(y)-\widetilde{B}\left(z_{1}, z_{2}\right)\right),
\end{aligned}
$$

and

$$
\begin{aligned}
& \widetilde{K}\left(z_{1}, z_{2}\right)=\left[\frac{\mu_{1} z_{2}+\mu_{2} z_{1}+\lambda z_{1} z_{2}}{\alpha}\right] \beta^{*}(y) \\
& \widetilde{A}\left(z_{1}, z_{2}\right)=\frac{\mu_{1}^{*}+\lambda z_{1}}{\lambda+\mu_{1}^{*}} \beta^{*}(y), \quad \widetilde{B}\left(z_{1}, z_{2}\right)=\frac{\mu_{2}^{*}+\lambda z_{2}}{\lambda+\mu_{2}^{*}} \beta^{*}(y) .
\end{aligned}
$$

In order to present the essential character of the analysis and to avoid intricate technical problems, we consider the following assumption: In the following, assume that $\mu_{1}^{*}=\mu_{1}+$ $\mu_{2}=\mu_{2}^{*}$. More precisely, when orbit queue 1 (resp. 2) empties, the re-dispatch rate at orbit queue 2 (resp. 1) increases to $\mu_{1}+\mu_{2}$. Taking into account that assumption, the functional Eq. (6.1) becomes,

$$
\begin{aligned}
& {\left[z_{1} z_{2}-\left(\bar{\mu}_{1} z_{2}+\bar{\mu}_{2} z_{1}\right) \check{\beta}^{*}\left(z_{1}, z_{2}\right)\right] \Pi\left(z_{1}, z_{2}\right)=\check{\beta}^{*}\left(z_{1}, z_{2}\right)} \\
& \quad \times\left\{\left(z_{2}-z_{1}\right)\left[\bar{\mu}_{2} \Pi\left(z_{1}, 0\right)-\bar{\mu}_{1} \Pi\left(0, z_{2}\right)\right]+\left[z_{1} z_{2}-\left(\bar{\mu}_{1} z_{1}+\bar{\mu}_{2} z_{2}\right)\right] \Pi(0,0)\right\},
\end{aligned}
$$

where $\bar{\mu}_{i}=\frac{\mu_{i}}{\mu_{1}+\mu_{2}}, i=1,2, \bar{\mu}=\mu_{1}+\mu_{2}$ and

$$
\check{\beta}^{*}\left(z_{1}, z_{2}\right)=\frac{\bar{\mu} \beta^{*}\left(\lambda\left(1-r_{1} z_{1}-r_{2} z_{2}\right)\right)}{\bar{\mu}+\lambda\left(1-\beta^{*}\left(\lambda\left(1-r_{1} z_{1}-r_{2} z_{2}\right)\right)\right)} .
$$

We have to note that $\check{\beta}^{*}\left(z_{1}, z_{2}\right)$ is a probability generating function. Indeed, let $\check{B}$ be the time elapsed from the epoch a service is initiated until the epoch the server remains idle just after a service completion of an orbiting customer of either type. Then, it is easy to see that the probability generating function of the number of customers that arrive during $\check{B}$ is

$$
\check{\beta}^{*}\left(z_{1}, z_{2}\right)=\sum_{k=0}^{\infty}\left(\frac{\lambda}{\lambda+\bar{\mu}}\right)^{k} \frac{\bar{\mu}}{\lambda+\bar{\mu}}\left(\beta^{*}\left(\lambda\left(1-r_{1} z_{1}-r_{2} z_{2}\right)\right)\right)^{k+1} .
$$


Remark 1: Note that the model under generally distributed service requirements, is closely connected with a two queue polling system with probabilistic scheduling and a generalized service time. More precisely, the server offers service of duration $\check{B}$, and upon such a service completion epoch, the next customer to be served comes from queue $i$ with probability $\bar{\mu}_{i}$, $i=1,2$.

The analysis of the kernel of $(6.5), z_{1} z_{2}-\left(\bar{\mu}_{1} z_{2}+\bar{\mu}_{2} z_{1}\right) \check{\beta}^{*}\left(z_{1}, z_{2}\right)$ is the starting point for the determination of $\Pi\left(z_{1}, z_{2}\right)$, which is regular for $\left|z_{1}\right|<1$, continuous for $\left|z_{1}\right| \leq 1$ for every fixed $z_{2}$ with $\left|z_{2}\right| \leq 1$; and similarly, with $z_{1}, z_{2}$ interchanged.

\subsection{Analysis of the Kernel}

Following Cohen and Boxma [17], consider the kernel for

$$
z_{1}=g s, z_{2}=g s^{-1},|s|=1,|g| \leq 1 .
$$

Consequently,

$$
\begin{aligned}
& \widehat{K}\left(g s, g s^{-1}\right)=g^{2}-g\left[\bar{\mu}_{1} s^{-1}+\bar{\mu}_{2} s\right] \check{\beta}^{*}\left(g s, g s^{-1}\right) \\
& \check{\beta}^{*}\left(g s, g s^{-1}\right)=\frac{\bar{\mu} \beta^{*}\left(\lambda\left(1-g\left(r_{1} s+r_{2} s^{-1}\right)\right)\right.}{\bar{\mu}+\lambda\left(1-\beta^{*}\left(\lambda\left(1-g\left(r_{1} s+r_{2} s^{-1}\right)\right)\right)\right.} .
\end{aligned}
$$

LEMMA 5:

1. If $\lambda \bar{b}\left(\frac{\bar{\mu}+\lambda}{\bar{\mu}}\right)<1$ the kernel $\widehat{K}\left(g_{s}, g^{-1}\right)$ has in $|g| \leq 1$ exactly two zeros, of which one is identically zero. The other zero, say $g=g(s)$, is given by

$$
g(s)=E\left(\left[r_{1} s+r_{2} s^{-1}\right]^{n-1}\left[\bar{\mu}_{1} s^{-1}+\bar{\mu}_{2} s\right]^{n}\right),
$$

where $n$ be the number of customers being served in a busy period of an $M / G / 1$ queue with arrival rate $\lambda$ and service time distribution with LST $\check{\beta}^{*}$.

2. For $|s|=1$,

$$
g(s)=-g(-s), \quad g(\bar{s})=\overline{g(s)}
$$

Proof: See the appendix.

Define,

$$
S_{1}:=\left\{z_{1}: z_{1}=g(s) s,|s|=1\right\}, \quad S_{2}:=\left\{z_{2}: z_{2}=g(s) s^{-1},|s|=1\right\} .
$$

A point of major concern is to prove that the contours $S_{1}, S_{2}$ are simply connected. However, this is not true for general $\bar{\mu}_{i}, r_{i}$. We further restrict our analysis to cases where it is rather easily to prove that $S_{1}, S_{2}$ are simply connected, and from hereon we will also assume that:

$$
\bar{\mu}_{1}=r_{1}, \quad \bar{\mu}_{2}=r_{2}, \quad r_{1}>r_{2} .
$$

Under that assumption it follows that

$$
g(s)=\left[r_{1} s^{-1}+r_{2} s\right] E\left(\left|r_{1} s+r_{2} s^{-1}\right|^{2 n-2}\right),
$$

since, for $|s|=1, r_{1} s^{-1}+r_{2} s=\overline{r_{1} s+r_{2} s^{-1}}$. 
Lemma 6: The contours $S_{1}, S_{2}$ are simply connected and smooth.

Proof: See the appendix.

It is easily seen that $z_{1}=0 \in S_{1}^{-}, z_{2}=0 \in S_{2}^{+}$, where $S_{i}^{+}, S_{i}^{-}$denote the interior and exterior of the contour $S_{i}, i=1,2$, respectively.

\subsection{Kernel's Parametrization}

Now, following Cohen and Boxma [17], p. 161, we have to consider the following boundary value problem for the functions $z_{1}:=g(s) s, z_{2}:=g(s) s^{-1}$ : To construct in the $x$-plane a smooth contour $L$ and a pair of mappings $z_{1}(x), x \in L^{+} \cup L, z_{2}(x), x \in L^{-} \cup L$ such that:

1. $z_{1}(x)$ is regular and univalent for $x \in L^{+}$, continuous for $x \in L^{+} \cup L$;

$z_{2}(x)$ is regular and univalent for $x \in L^{-}$, continuous for $x \in L^{-} \cup L$.

2. $z_{1}(x)$ maps $L^{+}$conformally onto $S_{1}^{+}$;

$z_{2}(x)$ maps $L^{-}$conformally onto $S_{2}^{+}$.

3. $z_{1}^{+}(x), z_{2}^{-}(x), x \in L$ is a zero pair of (6.2), where

$$
z_{1}^{+}(x)=\lim _{y \rightarrow x, y \in L^{+}} z_{1}(y), \quad z_{2}^{-}(x)=\lim _{y \rightarrow x, y \in L^{-}} z_{2}(y) .
$$

4. $z_{1}(0)>0, z_{1}(1)=1, z_{2}(\infty)=0,0<d:=\lim _{|x| \rightarrow \infty}\left|x z_{2}(x)\right|<\infty$.

We proceed with the determination of $L$, and the mappings $z_{1}(),. z_{2}($.$) . Since for x \in L$, $\left(z_{1}^{+}(x), z_{2}^{-}(x)\right)$ is a zero pair of $(6.2)$ for $\left|z_{1}\right| \leq 1,\left|z_{2}\right| \leq 1$ with $z_{1}^{+}(x) \in S_{1}, z_{2}^{-}(x) \in S_{2}$, we may write

$$
z_{1}^{+}(x)=g\left(e^{i \lambda(x)}\right) e^{i \lambda(x)}, \quad z_{2}^{-}(x)=g\left(e^{i \lambda(x)}\right) e^{-i \lambda(x)},
$$

where $\lambda():. L \rightarrow[0, \pi], \lambda(1)=0$. Then it is seen that for $x \in L$ :

$$
\begin{aligned}
& \log z_{1}^{+}(x)+\log \frac{x z_{2}^{-}(x)}{d}=\log \frac{g^{2}\left(e^{i \lambda(x)}\right)}{d}+\log x, \\
& \log z_{1}^{+}(x)-\log \frac{x z_{2}^{-}(x)}{d}=2 i \lambda(x)-\log x+\log d .
\end{aligned}
$$

Using the usual procedure as in [17], the solution of the above boundary value problem is:

$$
\begin{aligned}
& z_{1}(x)=\exp \left\{\frac{1}{2 \pi i} \int_{\zeta \in L}\left[\log \left\{g\left(e^{i \lambda(\zeta)}\right) \zeta^{1 / 2}\right\}\right]\left\{\frac{\zeta+x}{\zeta-x}-\frac{\zeta+1}{\zeta-1} \frac{d \zeta}{\zeta}\right\}\right\}, \quad x \in L^{+}, \\
& z_{2}(x)=x^{-1} \exp \left\{-\frac{1}{2 \pi i} \int_{\zeta \in L}\left[\log \left\{g\left(e^{i \lambda(\zeta)}\right) \zeta^{1 / 2}\right\}\right]\left\{\frac{\zeta+x}{\zeta-x}-\frac{\zeta+1}{\zeta-1} \frac{d \zeta}{\zeta}\right\}\right\}, \quad x \in L^{-} .
\end{aligned}
$$

Applying Plemelji-Sokhotski formulas for $x \in L$ gives:

$$
\begin{aligned}
& z_{1}^{+}(x)=g\left(e^{i \lambda(x)}\right) x^{1 / 2} \exp \left\{\frac{1}{2 \pi i} \int_{\zeta \in L}\left[\log \left\{g\left(e^{i \lambda(\zeta)}\right) \zeta^{1 / 2}\right\}\right]\left\{\frac{\zeta+x}{\zeta-x}-\frac{\zeta+1}{\zeta-1} \frac{d \zeta}{\zeta}\right\}\right\}, \\
& z_{2}^{-}(x)=g\left(e^{i \lambda(x)}\right) x^{-1 / 2} \exp \left\{-\frac{1}{2 \pi i} \int_{\zeta \in L}\left[\log \left\{g\left(e^{i \lambda(\zeta)}\right) \zeta^{1 / 2}\right\}\right]\left\{\frac{\zeta+x}{\zeta-x}-\frac{\zeta+1}{\zeta-1} \frac{d \zeta}{\zeta}\right\}\right\} .
\end{aligned}
$$


Substituting back in (6.10) gives the following relation for the determination of $L, \lambda(x)$, $x \in L$ :

$$
\exp \{i \lambda(x)\}=x^{1 / 2} \exp \left\{\frac{1}{2 \pi i} \int_{\zeta \in L}\left[\log \left\{g\left(e^{i \lambda(\zeta)} \zeta^{1 / 2}\right\}\right]\left\{\frac{\zeta+x}{\zeta-x}-\frac{\zeta+1}{\zeta-1} \frac{d \zeta}{\zeta}\right\}\right\} .\right.
$$

\subsection{Solution of a Riemann Boundary Value Problem}

Since for $x \in L,\left(z_{1}^{+}(x), z_{2}^{-}(x)\right)$ is a zero pair of (6.2), it readily follows from (6.5) that

$$
\begin{aligned}
& r_{2}\left(z_{1}^{+}(x)-z_{2}^{-}(x)\right) \Pi\left(z_{1}^{+}(x), 0\right)+r_{1}\left(z_{2}^{-}(x)-z_{1}^{+}(x)\right) \Pi\left(0, z_{2}^{-}(x)\right) \\
& \quad=\left(z_{1}^{+}(x) z_{2}^{-}(x)-r_{1} z_{1}^{+}(x)-r_{2} z_{2}^{-}(x)\right) \Pi(0,0) .
\end{aligned}
$$

Using (6.9) we conclude in:

$$
\Pi\left(z_{1}^{+}(x), 0\right)=\frac{r_{1}}{r_{2}} \Pi\left(0, z_{2}^{-}(x)\right)+\widehat{J}(x)
$$

where

$$
\widehat{J}(x)=\frac{g\left(e^{i \lambda(x)}\right)-r_{1} e^{i \lambda(x)}-r_{2} e^{-i \lambda(x)}}{r_{2}\left(r_{1} e^{i \lambda(x)}-r_{2} e^{-i \lambda(x)}\right)} \Pi(0,0) .
$$

Thus, we come up with the following Riemann boundary value problem: For a simply connected Jordan contour $L$ and the mappings $z_{1}(x), x \in L \cup L^{+}, z_{2}(x), x \in L \cup L^{-}$, find two functions such that

1. $\Pi\left(z_{1}(x), 0\right)$ is regular for $x \in L^{+}$, continuous for $x \in L \cup L^{+}$; $\Pi\left(0, z_{2}(x)\right)$ is regular for $x \in L^{-}$, continuous for $x \in L \cup L^{-}$.

2. Satisfying the boundary condition: for $x \in L$,

$$
\Pi\left(z_{1}^{+}(x), 0\right)=\frac{r_{1}}{r_{2}} \Pi\left(0, z_{2}^{-}(x)\right)+\widehat{J}(x) .
$$

Since the $\frac{r_{1}}{r_{2}}$ never vanishes, its index is ind $x \in L \frac{r_{1}}{r_{2}}=0$, and clearly $\frac{r_{1}}{r_{2}}$ satisfies the Holder condition on $L$. Moreover, $\widehat{J}(x)$ satisfies the Holder condition on $L$, since both its numerator and its denominator satisfies it. Thus, the solution of this Riemann boundary value problem is given by:

$$
\begin{array}{ll}
r_{2} \Pi\left(z_{1}(x), 0\right)=\frac{1}{2 i \pi} \int_{\zeta \in L} \widehat{J}(\zeta) \frac{d \zeta}{\zeta-x}+r_{1} \Pi(0,0), & x \in L^{+}, \\
r_{1} \Pi\left(0, z_{2}(x)\right)=\frac{1}{2 i \pi} \int_{\zeta \in L} \widehat{J}(\zeta) \frac{d \zeta}{\zeta-x}+r_{2} \Pi(0,0), & x \in L^{-}
\end{array}
$$

Let now $x=w_{1}\left(z_{1}\right), z_{1} \in S_{1}^{+}$, and $x=w_{2}\left(z_{2}\right), z_{2} \in S_{2}^{+}$the inverse mappings of $z_{1}(x)$, $z_{2}(x)$, respectively. These are conformal mappings of $S_{1}^{+}$(resp. $S_{2}^{+}$) onto $L^{+}$(resp. $L^{-}$). Since $S_{1}, S_{2}$ and $L$ are smooth, by the theorem of corresponding boundaries [47] is implied that $w_{1}($.$) maps S_{1}$ onto $L$, and $w_{2}($.$) maps S_{2}$ onto $L$. Finally, let $\omega_{2}: S_{1} \rightarrow S_{2}$ a mapping from the contour $S_{1}$ onto the contour $S_{2}$ (its existence is ensured due to Theorem 1.1, 
p. 101 in [19]), such that, for every $x \in L$, the pair $\left(z_{1}^{+}(x), \omega_{2}\left(z_{1}^{+}(x)\right)\right)$ is a zero pair of the kernel (6.2), that is, $\omega_{2}\left(z_{1}^{+}(x)\right)=z_{2}^{-}(x)$. Then, $\widehat{J}(x)$ may be rewritten as:

$$
\widehat{J}(x)=\frac{z_{1}^{+}(x) \omega_{2}\left(z_{1}^{+}(x)\right)-r_{1} z_{1}^{+}(x)-r_{2} \omega_{2}\left(z_{1}^{+}(x)\right)}{z_{1}^{+}(x)-\omega_{2}\left(z_{1}^{+}(x)\right)} \Pi(0,0)=f(x) \Pi(0,0) .
$$

Applying the Plemelj-Sokhotski formulas [33] we have for $x \in L$

$$
\begin{aligned}
& r_{2} \Pi\left(z_{1}^{+}(x), 0\right)=\frac{\Pi(0,0)}{2 i \pi} \int_{\zeta \in L} f(\zeta) \frac{d \zeta}{\zeta-x}+\frac{1}{2} f(x) \Pi(0,0)+r_{1} \Pi(0,0), \\
& r_{1} \Pi\left(0, z_{2}^{-}(x)\right)=\frac{\Pi(0,0)}{2 i \pi} \int_{\zeta \in L} f(\zeta) \frac{d \zeta}{\zeta-x}-\frac{1}{2} f(x) \Pi(0,0)+r_{2} \Pi(0,0) .
\end{aligned}
$$

We now obtain $\Pi(0,0)$, by deriving some preliminary results from the functional Eq. (6.5), under assumption (6.8). Setting $z_{2}=1$ in (6.5), and subsequently letting $z_{2} \rightarrow 1$, and vice versa yields the following linear relations between $\Pi(0,1), \Pi(1,0)$ and $\Pi(0,0)$ :

$$
\begin{aligned}
& r_{1}(1-\rho)=r_{1} \Pi(0,1)-r_{2} \Pi(1,0)+r_{2} \Pi(0,0), \\
& r_{2}(1-\rho)=r_{2} \Pi(1,0)-r_{1} \Pi(0,1)+r_{1} \Pi(0,0),
\end{aligned}
$$

Summing (6.13), results in $\Pi(0,0)=1-\rho$. Letting $x \rightarrow 1$ in the first of (6.11) we obtain $\Pi(1,0)$ and then substituting in one from $(6.13)$ we can obtain $\Pi(0,1)$. Note that $\Pi(1,0)$, $\Pi(0,1)$ denote the probability of finding the second and the first orbit queue empty at service completion epochs, respectively.

Note also that the stability condition $\rho=\lambda \bar{b}\left(\frac{\bar{\mu}+\lambda}{\bar{\mu}}\right)<1$ has a very nice probabilistic interpretation. Indeed,

$$
\rho=\lambda \bar{b}\left(\frac{\bar{\mu}+\lambda}{\bar{\mu}}\right)<1 \Leftrightarrow \lambda \bar{b}<1-\frac{\lambda}{\bar{\mu}+\lambda} .
$$

This condition can be rewritten as

$$
\begin{aligned}
E\left(B_{n}^{(1)}=\right. & \left.1 \mid X_{i}(n)>0, i=1,2\right)+E\left(B_{n}^{(2)}=1 \mid X_{i}(n)>0, i=1,2\right) \\
& +E\left(A_{1}(n)\right)+E\left(A_{2}(n)\right)<1,
\end{aligned}
$$

where $E\left(A_{i}(n)\right)=\lambda_{i} \bar{b}$ be the expected number of customers of type $i$ that arrive during a service time. If $B_{n}^{(i)}$ be the number of external arrivals of type $i$ at the epoch at which the $n$th service starts, then (6.14) says that the mean number of external arrivals of either type between two consecutive departures during a busy period must be smaller than one, which is expected in order the system to be stable.

\subsection{Performance Metrics and Computational Issues}

In the following, we derive expressions for $E\left(X_{i}\right)$, that is, the expected number of customers in orbit $i, i=1,2$. Using (6.5) for $z_{2}=1$ and differentiating with respect to $z_{1}$, and then 
setting $z_{1}=1$ and vice versa yields:

$$
\begin{aligned}
& E\left(X_{1}\right)=r_{1}\left[\rho+\frac{\lambda_{1}}{2(1-\rho)}\left(\frac{\bar{\mu}+\lambda}{\bar{\mu}}\right)\left(\bar{b}^{(2)}+\frac{\lambda(\bar{b})^{2}}{\bar{\mu}}\right)\right]-\left.\frac{r_{2}}{r_{1}(1-\rho)} \frac{d}{d z_{1}} \Pi\left(z_{1}, 0\right)\right|_{z_{1}=1}, \\
& E\left(X_{2}\right)=r_{2}\left[\rho+\frac{\lambda_{2}}{2(1-\rho)}\left(\frac{\bar{\mu}+\lambda}{\bar{\mu}}\right)\left(\bar{b}^{(2)}+\frac{\lambda(\bar{b})^{2}}{\bar{\mu}}\right)\right]-\left.\frac{r_{1}}{r_{2}(1-\rho)} \frac{d}{d z_{2}} \Pi\left(0, z_{2}\right)\right|_{z_{2}=1} .
\end{aligned}
$$

Differentiating (6.12) with respect to $x$ and substituting $x=1$ yields

$$
\begin{aligned}
\left.\frac{d}{d z_{1}} \Pi\left(z_{1}, 0\right)\right|_{z_{1}=1}= & \frac{1}{r_{2}\left(z_{1}^{+}\right)^{\prime}(1)}\left[\left.\frac{d}{d x}\left\{\frac{1-\rho}{2 i \pi} \int_{\zeta \in L} f(\zeta) \frac{d \zeta}{\zeta-x}\right\}\right|_{x=1}+\frac{1}{2}(1-\rho) f^{\prime}(1)\right] \\
& =\frac{1}{r_{2}\left(z_{1}^{+}\right)^{\prime}(1)}\left[\frac{1-\rho}{2 i \pi} \int_{\zeta \in L} \frac{f(\zeta)+f^{\prime}(1)}{\zeta-1} d \zeta\right] \\
\left.\frac{d}{d z_{2}} \Pi\left(0, z_{2}\right)\right|_{z_{2}=1} & =\frac{1}{r_{1} \omega_{2}^{\prime}(1)\left(z_{1}^{+}\right)^{\prime}(1)}\left[\left.\frac{d}{d x}\left\{\frac{1-\rho}{2 i \pi} \int_{\zeta \in L} f(\zeta) \frac{d \zeta}{\zeta-x}\right\}\right|_{x=1}-\frac{1}{2}(1-\rho) f^{\prime}(1)\right] \\
& =\frac{1}{r_{1} \omega_{2}^{\prime}(1)\left(z_{1}^{+}\right)^{\prime}(1)}\left[\frac{1-\rho}{2 i \pi} \int_{\zeta \in L} \frac{f(\zeta)-f^{\prime}(1)}{\zeta-1} d \zeta\right]
\end{aligned}
$$

where "'" means first-order derivative. For an efficient computation of the integrals, we proceed with the following parametrization of the contour $S_{1}$ using the mapping $\phi:[0,2 \pi] \rightarrow C$,

$$
\phi(t)=g\left(e^{(-1 / 2) i t}\right) e^{(1 / 2) i t},
$$

and set $\eta(t):=w_{1}^{+}(\phi(t)), t \in[0,2 \pi]$, where $w_{1}^{+}($.$) is the inverse mapping of z_{1}^{+}($.$) . Then,$

$$
f^{\prime}(1)=\left.\frac{1}{\eta^{\prime}(0)} \frac{d}{d t} f(\eta(t))\right|_{t=0}=\left.\frac{1}{\eta^{\prime}(0)} \frac{d}{d t}\left\{\frac{\phi(t) \omega_{2}(\phi(t))-r_{1} \phi(t)-r_{2} \omega_{2}(\phi(t))}{\phi(t)-\omega_{2}(\phi(t))}\right\}\right|_{t=0} .
$$

To proceed, we need some extra preliminary results. Having in mind that

$$
g(s)=\left(r_{1} s^{-1}+r_{2} s\right) \check{\beta}\left(g(s) s, g(s) s^{-1}\right),
$$

implicit differentiation with respect to $s$, at $s=1$ yields

$$
g^{\prime}(1)=r_{2}-r_{1}, \quad g^{\prime \prime}(1)=2 r_{1}\left(1+4 r_{2} \frac{\rho}{1-\rho}\right) .
$$

Thus, $\phi(0)=1, \phi^{\prime}(0)=r_{2} i, \phi^{\prime \prime}(1)=r_{2}\left(r_{1}-r_{2}-\frac{2 r_{1}}{1-\rho}\right)$. Since $\left(z_{1}^{+}(x), \omega_{2}\left(z_{1}^{+}(x)\right)\right), x \in L$ is a zero pair of the kernel, it is seen that

$$
z_{1}^{+}(x) \omega_{2}\left(z_{1}^{+}(x)=\left(r_{1} \omega_{2}\left(z_{1}^{+}(x)\right)+r_{2} z_{1}^{+}(x)\right) \check{\beta}\left(z_{1}^{+}(x), \omega_{2}\left(z_{1}^{+}(x)\right)\right),\right.
$$

and thus, differentiating with respect to $x$, at $x=1$ yields after some algebra

$$
\omega_{2}^{\prime}(1)=-\frac{r_{1}}{r_{2}}, \quad \omega_{2}^{\prime \prime}(1)=\frac{2 r_{1}}{r_{2}^{2}(1-\rho)} .
$$

Therefore,

$$
f^{\prime}(1)=\frac{r_{1} r_{2} i \rho}{(1-\rho) \eta^{\prime}(0)}, \quad\left(z_{1}^{+}\right)^{\prime}(1)=\frac{r_{2} i}{\eta^{\prime}(0)}
$$


Substituting the last equation into (6.16) yields

$$
\begin{aligned}
\left.\frac{d}{d z_{1}} \Pi\left(z_{1}, 0\right)\right|_{z_{1}=1} & =\frac{r_{1} \rho}{2 r_{2}}+\left.\frac{\eta^{\prime}(0)(1-\rho)}{r_{2}^{2} i} \frac{d}{d x}\left\{\frac{1}{2 i \pi} \int_{\zeta \in L} f(\zeta) \frac{d \zeta}{\zeta-x}\right\}\right|_{x=1}, \\
\left.\frac{d}{d z_{2}} \Pi\left(0, z_{2}\right)\right|_{z_{2}=1} & =\frac{r_{2} \rho}{2 r_{1}}-\left.\frac{\eta^{\prime}(0)(1-\rho)}{r_{1}^{2} i} \frac{d}{d x}\left\{\frac{1}{2 i \pi} \int_{\zeta \in L} f(\zeta) \frac{d \zeta}{\zeta-x}\right\}\right|_{x=1}
\end{aligned}
$$

Regarding the last term in the r.h.s. of (6.17) we write:

$$
\begin{aligned}
\left.\frac{d}{d x}\left\{\frac{1}{2 i \pi} \int_{\zeta \in L} f(\zeta) \frac{d \zeta}{\zeta-x}\right\}\right|_{x=1}= & \left.\frac{d}{d x}\left\{\frac{1}{2 i \pi} \int_{\zeta \in L} \frac{f(\zeta)-f(1)-(\zeta-1) f^{\prime}(1)}{\zeta-x} d \zeta\right\}\right|_{x=1} \\
& +\left.\frac{d}{d x}\left\{\frac{1}{2 i \pi} \int_{\zeta \in L} \frac{f(1)+(\zeta-1) f^{\prime}(1)}{\zeta-x} d \zeta\right\}\right|_{x=1} \\
= & \frac{1}{2 i \pi} \int_{\zeta \in L} \frac{f(\zeta)-f(1)-(\zeta-1) f^{\prime}(1)}{(\zeta-1)^{2}} d \zeta+\frac{1}{2} f^{\prime}(1) .
\end{aligned}
$$

Then, changing the variable of of integration we obtain:

$$
\left.\frac{d}{d x}\left\{\frac{1}{2 i \pi} \int_{\zeta \in L} f(\zeta) \frac{d \zeta}{\zeta-x}\right\}\right|_{x=1}=\frac{1}{2 i \pi} \int_{0}^{2 \pi} \frac{f(\eta(t))-f(1)-(\eta(t)-1) f^{\prime}(1)}{(\eta(t)-1)^{2}} \eta^{\prime}(t) d t+\frac{1}{2} f^{\prime}(1),
$$

with the aid of which we can found that $f(1)=r_{2}-r_{1}$. Substituting (6.17), (6.18) to the first equation in (6.15) we can obtain $E\left(X_{1}\right)$. Similarly we can obtain $E\left(X_{2}\right)$.

\section{NUMERICAL EXAMPLE}

In the following, we present a simple numerical result, focusing on the model with exponential service requirements analyzed in subsection 4.1. We investigate the probability of an empty system using (4.15) and assume that $\mu_{2}=3, \mu=4, \mu_{1}^{*}=3, \mu_{2}^{*}=4$. The contour integrals in (4.15) were evaluated by numerical integration using the trapezium method.

More precisely, we firstly change the variable $t=\sqrt{\widehat{\mu}_{1} / \widehat{\lambda_{1}}} e^{i \phi}, \phi \in[0,2 \pi)$, changing the differential to $d t=\sqrt{\widehat{\mu}_{1} / \widehat{\lambda_{1}}} i e^{i \phi} d \phi$. Then, we divided the interval $[0.2 \pi)$ into $K=20,000$ equal parts and apply the trapezium numerical integration approach, evaluating the integrand at points $\phi_{k}=2 \pi k / 20,000, k=0,1, \ldots, K-1$.

In Figure 2, we can observe the impact of $\lambda_{2}$ on the probability of an empty system, for different values of $\lambda_{1}$. Clearly, as we increase $\lambda_{2}, P\left(N_{1}=0, N_{2}=0, C=0\right)$ decreases, a result that is expected. Figures 3 and 4 compare $P\left(N_{1}=0, N_{2}=0, C=0\right)$ for our model and the model in Avrachenkov et al. [7], where no coupled orbits were considered. In Figure 3, we observe that when $\lambda_{2}$ takes relatively small values, $P\left(N_{1}=0, N_{2}=0, C=0\right)$ is clearly larger for our model. However, as $\lambda_{2}$ increases, the impact of coupled rates decreases, since it is more likely the arriving customers to occupy the server, and thus, the two systems give similar values on $P\left(N_{1}=0, N_{2}=0, C=0\right)$. Finally, Figure 4 describes the impact of coupled retrial rates on $P\left(N_{1}=0, N_{2}=0, C=0\right)$ for increasing values of $\mu_{1}$. It is seen that the increase in $\mu_{1}$, will increase $P\left(N_{1}=0, N_{2}=0, C=0\right)$, and clearly for the model with coupled rates, that increase is more apparent, compared with the model in [7]. 


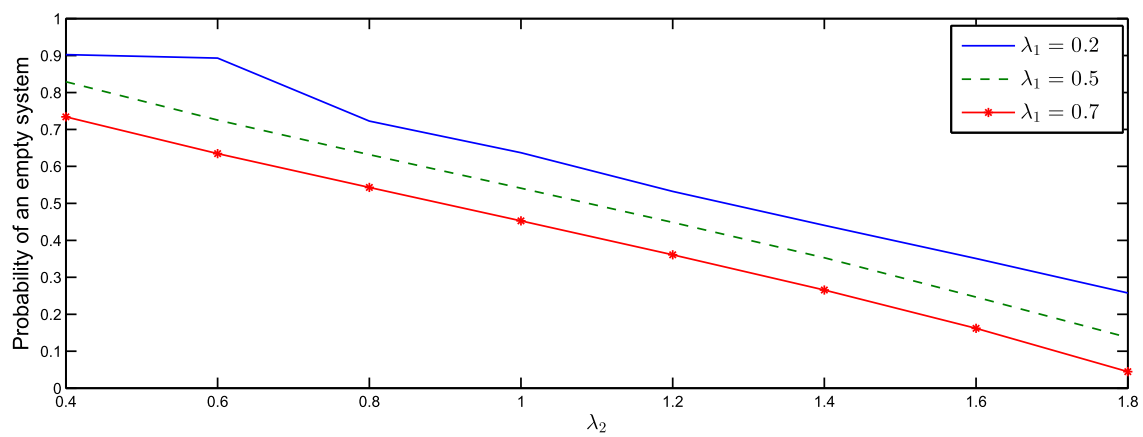

Figure 2. Probability of an empty system for $\mu_{1}=2$.

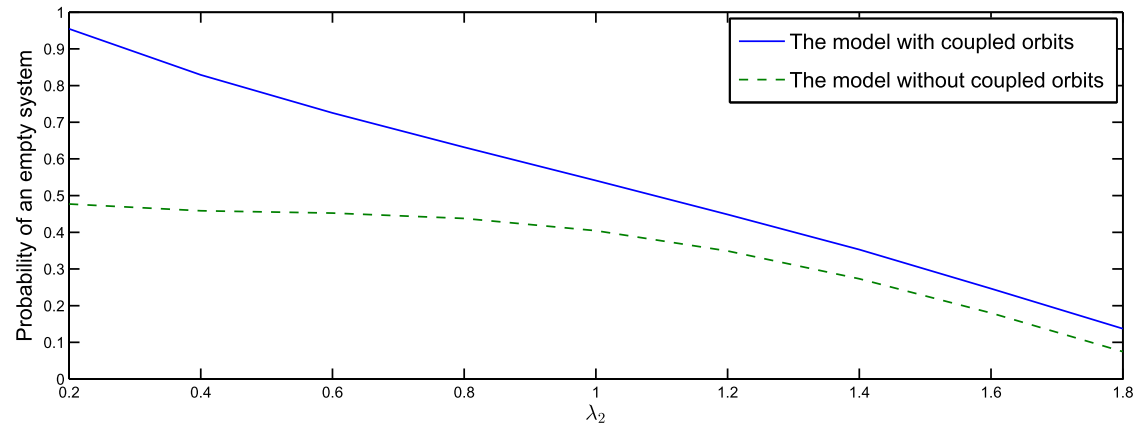

Figure 3. Comparison between our model and the model in [7] for $\lambda_{1}=0.5, \mu_{1}=2$.

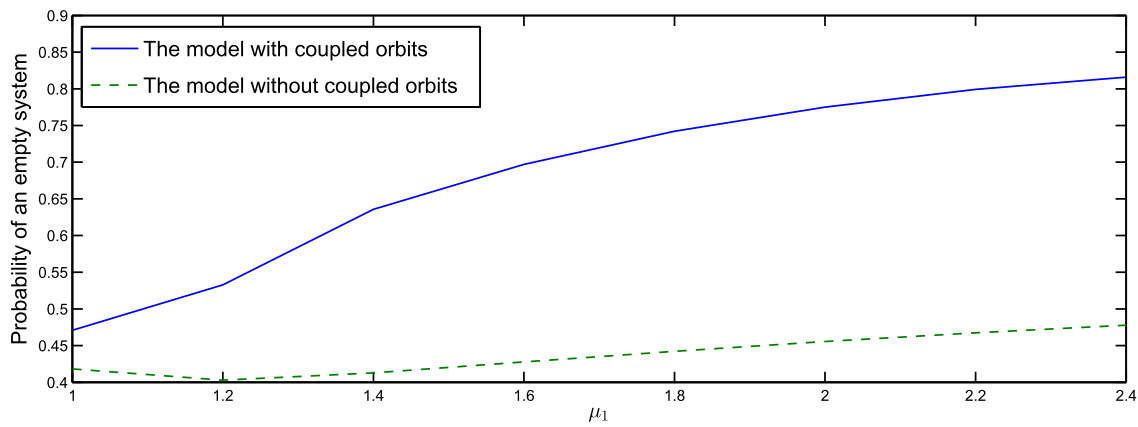

Figure 4. Comparison between our model and the model in [7] for $\lambda_{1}=0.5=\lambda_{2}$.

\section{CONCLUSION}

In this paper, we provided a thorough investigation of retrial queueing systems with two classes of retrial customers and coupled orbit queues. The main characteristic of the system is that when an orbit queue empties, the re-transmission rate of the other one is affected. For exponential service requirements, stability conditions were investigated and the joint orbit queue length distribution was obtained with the aid of Riemann-Hilbert boundary value theory. Exact tail asymptotic properties of the stationary orbit queue length distribution were also investigated. For arbitrary distributed service times, the joint orbit queue length distribution was obtained in terms of the solution of a non-homogeneous Riemann boundary 
value theory. Performance metrics were derived and used to present a simple numerical example.

\section{Acknowledgments}

The author is grateful to the Editor and the anonymous referee for the valuable remarks and comments, from which the presentation of the paper has benefited.

\section{References}

1. Adan, I., Boxma, O., Kapodistria, S., \& Kulkarni, V. (2016). The shortest queue polling model. Annals of Operations Research 241(1): 167-200.

2. Andradottir, S., Ayhan, H., \& Down, D. (2001). Server assignment policies for maximizing the steadystate throughput of finite queueing systems. Management Science 47: 1421-1439.

3. Artalejo, J.R. (1999). A classified bibliography of research on retrial queues: progress in 1990-1999. Top 7: $187-211$.

4. Artalejo, J.R. (1999). Accessible bibliography on retrial queues. Mathematical and Computer Modeling 30: $1-6$.

5. Artalejo, J.R. \& Gomez-Corral, A. (2008). Retrial queueing systems: a computational approach. Berlin: Springer-Verlag.

6. Artalejo, J.R. (1997). Analysis of an M/G/1 queue with constant repeated attempts and server vacations. Computers and Operations Research 24(6): 493-504.

7. Avrachenkov, K., Nain, P., \& Yechiali, U. (2014). A retrial system with two input streams and two orbit queues. Queueing Systems 77: 1-31.

8. Avrachenkov, K. \& Yechiali, U. (2010). Retrial networks with finite buffers and their applications to internet data traffic. Probability in the Engineering and Informational Sciences 22: 519-536.

9. Badila, S. \& Resing, J.A.C. (2016). A coupled processor model with simultaneous arrivals and ordered service requirements. Queueing Systems 82: 29-42.

10. Borst, S., Boxma, O., \& Uitert, M. (2003). The asymptotic workload behavior of two coupled queues. Queueing Systems 43(1-2): 81-102.

11. Borst, S., Boxma, O., \& Jelenkovic, P. (2000). Coupled processors with regularly varying service times. In INFOCOM 2000 Proceedings, IEEE, vol. 1, pp. 157-164.

12. Borst, S., Jonckheere, M., \& Leskela, L. (2008). Stability of parallel queueing systems with coupled service rates. Discrete Event Dynamic Systems 18(4): 447-472.

13. Borst, Hegde, S., \& Proutiere, A. (2009). Interacting queues with server selection and coordinated scheduling: application to cellular data networks. Annals of Operations Research 170(1): 59-78.

14. Boxma, O. \& Groenendijk, W.P. (1988). Two queues with alternating service and switching times. In O. Boxma \& R. Syski (eds.), Queueing theory and its applications. Amsterdam: North-Holland, pp. 261-281.

15. Boxma, O. (1984). Two symmetric queues with alternating service and switching times. In E. Gelenbe (ed.), Performance '84. Amsterdam: North-Holland, pp. 409-431.

16. Cohen, J.W. (1982). The single server queue. Amsterdam: North-Holland.

17. Cohen, J.W. \& Boxma, O. (1983). Boundary value problems in queueing systems analysis. Amsterdam: North-Holland.

18. Cohen, J.W. (1987). A two queue, one server model with priority for the longer queue. Queueing Systems 2: 261-283.

19. Cohen, J.W. (1988). Boundary value problems in queueing theory. Queueing Systems 3: 97-128.

20. Cohen, J.W. (1992). Analysis of random walks. Amsterdam: I.O.S. Press.

21. Choi, B.D., Park, K.K., \& Pearce, C.E.M. (1993). The M/M/1 retrial queue with control policy and general retrial times. Queueing Systems 14: 275-292.

22. Choi, B.D., Rhee, K. H., \& Park, K.K. (1993). The M/G/1 retrial queue with retrial rate control policy. Probability in the Engineering and Informational Sciences 7: 29-46.

23. Denteneer, D. \& van Leeuwaarden, J.S.H. (2008). Multiaccess, Reservations \& Queues. Philips Research Book Series, vol. 10, Heidelberg, Berlin: Springer-Verlag.

24. Dimitriou, I. (2016). A queueing model with two types of retrial customers and paired services. Annals of Operations Research 238(1): 123-143.

25. Falin, G.I. \& Templeton, J.G.C. (1997). Retrial queues. London: Chapman \& Hall. 
26. Falin, G.I. (1988). On a multiclass batch arrival retrial queue. Advances in Applied Probability 20: 483-487.

27. Farahmand, K. (1990). Single line queue with repeated demands. Queueing Systems 6: 223-228.

28. Fayolle, G. (1986). A simple telephone exchange with delayed feedbacks. In O.J. Boxma, J.W. Cohen \& M.C. Tijms (eds.), Proceedings of the International Conference on Teletraffic Analysis and Computer Performance Evaluation. Amsterdam: North-Holland, pp. 245-253.

29. Fayolle, G. \& Iasnogorodski, R. (1979). Two coupled processors: The reduction to a Riemann-Hilbert problem. Zeitschrift fur Wahrscheinlichkeitstheorie und Verwandte Gebiete 47: 325-351.

30. Fayolle, G., Iasnogorodski, R., \& Malyshev, V. (1999). Random walks in the quarter-plane, algebraic methods, boundary value problems and applications. Vol. 40 of Applications of Mathematics. Berlin: Springer-Verlag.

31. Feng, W., Kowada, M., \& Adachi, K. (1998). A two-queue model with Bernoulli service schedule and switching times. Queueing Systems 30: 405-434.

32. Flajolet, F. \& Sedgewich, R. (2009). Analytic combinatorics. New York: Cambridge University Press.

33. Gakhov, F.D. (1966). Boundary value problems. Oxford: Pergamon Press.

34. Grishechkin, S.A. (1992). Multiclass batch arrival retrial queues analyzed as branching processes with immigration. Queueing Systems 11: 395-418.

35. Guillemin, F. \& van Leeuwaarden, J.H.S. (2011). Rare event asymptotics for a random walk in the quarter plane. Queueing Systems 67: 1-32.

36. Guillemin, F. \& Pinchon, D. (2004). Analysis of generalized processor-sharing systems with two classes of customers and exponential services. Journal of Applied Probability 41: 832-858.

37. Hunter, T.E. \& Nosratinia, A. (2004). Diversity through coded cooperation. IEEE Transactions on Wireless Communications 5: 283-289.

38. Janssens, G.K. (1997). The quasi-random input queueing system with repeated attempts as a model for a collision-avoidance star local area network. IEEE Transactions on Communications 45: 360-364.

39. Khomichkov, I.I. (1995). Calculation of the characteristics of local area network with p-persistent protocol of multiple random access. Automation and Remote Control 56: 208-218.

40. Kulkarni, V.G. (1986). Expected waiting times in a multiclass batch arrival retrial queue. Journal of Applied Probability 23: 144-154.

41. Langaris C. \& Dimitriou, I. (2010). A queueing system with n-phases of service and (n-1)-types of retrial customers. European Journal of Operations Research 205: 638-649.

42. Li, H. \& Zhao, Y.Q. (2011). Tail asymptotics for a generalized two-demand queuing model-a kernel method. Queueing Systems 69: 77-100.

43. Li, H. \& Zhao, Y.Q. (2015). A kernel method for exact tail asymptotics for random walks in the quarter plane. http://arxiv.org/pdf/1505.04425v1.pdf

44. Moutzoukis, E. \& Langaris, C. (1996). Non-preemptive priorities and vacations in a multiclass retrial queueing system. Stochastic Models 12(3): 455-472.

45. Muskhelishvili, N.I. (1992). Singular integral equations. New York: Dover Publications.

46. Nain, P. (1985). Analysis of a two-node Aloha network with infinite capacity buffers. In T. Hasegawa, H. Takagi \& Y. Takahashi (eds.), Proceedings of the International Seminar on Computer Networking and Performance Evaluation. Tokyo, Japan, pp. 49-63.

47. Nehari, Z. (1975). Conformal mapping. New York: Dover Publications.

48. Papadimitriou, G., Pappas, N., Traganitis, A., \& Angelakis, V. (2015). Network-level performance evaluation of a two-relay cooperative random access wireless system. Computer Networks 88: $187-201$.

49. Pappas, N., Kountouris, M., Ephremides, A., \& Traganitis, A. (2015). Relay-assisted multiple access with full-duplex multi-packet reception. IEEE Transactions on Wireless Communications 14: 35443558 .

50. Resing, J.A.C. \& Ormeci, L. (2003). A tandem queueing model with coupled processors. Operations Research Letters 31: 383-389.

51. Rengarajan, B., Caramanis, C., \& De Veciana, G. (2008). Analyzing queueing systems with coupled processors through semi definite programming. INFORMS: Applied Probability Session, http://users.ece.utexas.edu/gustavo/papers/SdpCoupledQs.pdf

52. Song, Y., Liu, Z., \& Zhao, Y. (2016). Exact tail asymptotics: revisit of a retrial queue with two input streams and two orbits. Annals of Operations Research 247: 97-120.

53. Szpankowski, W. (1994). Stability conditions for some multiqueue distributed systems: Buffered random access systems. Advances in Applied Probability 26: 498-515.

54. Takahashi, Y. (1990). Coupled processor: a second order continuous state model. Probability in the Engineering and Informational Sciences 4: 277-298. 
55. Van Leeuwaarden, J.S.H., \& Resing, J.A.C. (2005). A tandem queue with coupled processors: Computational issues. Queueing Systems 50: 29-52.

56. Vitale, C., Rizzo, G., Rengarajan, B., \& Mancuso, V. (2015). An analytical approach to performance analysis of coupled processor systems. ITC 2015 Proceedings of 27 th International Teletraffic Congress, IEEE, Ghent, Belgium, pp. 89-97.

57. Vitale, C, Rizzo, G., \& Mancuso, V. (2015). A coupled processors model for 802.11 ad hoc networks under non saturation. In ICC 2015, IEEE International Conference on Communications, London, UK, pp. 628-634.

58. Vitale, C, Mancuso, V., \& Rizzo, G. (2015). Modelling D2D communications in cellular access networks via coupled processors. COMSNETS 2015 Proceedings of 7 th International Conference on Communication Systems and Networks, Bangalore, India, pp. 1-8.

\section{APPENDIX}

Lemma 7: Under stability condition, $B\left(X_{0}(y), y\right) \neq 0, y \in\left[y_{1}, y_{2}\right]$.

Proof: $K(x, y)=0$ reads,

$$
y(x-1)\left(\widehat{\mu}_{1}-\widehat{\lambda}_{1} x\right)=x(y-1)\left(\widehat{\lambda}_{2} y-\widehat{\mu}_{2}\right) .
$$

Combining with $B(x, y)=0$, yields for $(x, y) \notin\{(0,0),(1,1)\}$ :

$$
\lambda_{2} \mu_{1}\left(\lambda+\mu_{2}-d_{2}\right) y+\lambda_{1}\left(\mu_{2} \mu_{1}-d_{2}\left(\lambda+\mu_{1}\right) x-\widehat{\mu}_{2} \mu_{1}+\widehat{\mu}_{1} d_{2}=0 .\right.
$$

However, for $|x| \leq 1,|y| \leq 1$,

$$
\begin{aligned}
& \mid \lambda_{2} \mu_{1}\left(\lambda+\mu_{2}-d_{2}\right) y+\lambda_{1}\left(\mu_{2} \mu_{1}-d_{2}\left(\lambda+\mu_{1}\right) x-\widehat{\mu}_{2} \mu_{1}+\widehat{\mu}_{1} d_{2} \mid\right. \\
& \quad \leq \lambda_{2} \mu_{1}\left(\lambda+\mu_{2}-d_{2}\right)+\lambda_{1}\left(\mu_{2} \mu_{1}-d_{2}\left(\lambda+\mu_{1}\right) x-\widehat{\mu}_{2} \mu_{1}+\widehat{\mu}_{1} d_{2}\right. \\
& \quad=\left[\widehat{\mu}_{1}-\lambda\left(\lambda_{1}+\mu_{1}\right)\right]\left[d_{2}+\mu_{1} \frac{\lambda\left(\lambda_{2}+\mu_{2}\right)-\widehat{\mu}_{2}}{\widehat{\mu}_{1}-\lambda\left(\lambda_{1}+\mu_{1}\right)}\right]<0,
\end{aligned}
$$

under stability conditions. This proves that the only solutions to $K(x, y)=B(x, y)=0$ in $|x| \leq 1$, $|y| \leq 1$, are $(0,0),(1,1)$, and thus $B\left(X_{0}(y), y\right) \neq 0$ for $y \in\left[y_{1}, y_{2}\right]$.

Proof of Lemma 5: Clearly, for fixed $|s|=1, g=0$ is a zero of $K\left(g s, g s^{-1}\right),|g| \leq 1$. For $|g|=1$ it is seen that for $|s|=1, s \neq \pm 1$

$$
\left|\left[\bar{\mu}_{1} s^{-1}+\bar{\mu}_{2} s\right] \check{\beta}^{*}\left(g s, g s^{-1}\right)\right| \leq\left|\bar{\mu}_{1} s^{-1}+\bar{\mu}_{2} s\right|<1=|g| .
$$

Since $\breve{\beta}^{*}\left(g s, g s^{-1}\right)$ is regular in $g$ for $|g|<1$, with fixed $s,|s|=1$, and continuous for $|g| \leq 1$, by applying Rouche's theorem [16] to the contour $|g|=1$ it is seen that $g^{-1} K\left(g s, g s^{-1}\right)$ has for every fixed $s,|s|=1$ a unique zero in $|g| \leq 1$, with multiplicity 1 . Set now,

$$
h(s)=\left(r_{1} s+r_{2} s^{-1}\right) g .
$$

Substituting in

$$
g^{-1} K\left(g s, g s^{-1}\right)=g-\left[\bar{\mu}_{1} s^{-1}+\bar{\mu}_{2} s\right] \check{\beta}^{*}\left(g s, g s^{-1}\right),
$$

we conclude in

$$
h=\left[\bar{\mu}_{1} s^{-1}+\bar{\mu}_{2} s\right]\left[r_{1} s+r_{2} s^{-1}\right] \frac{\bar{\mu} \beta^{*}(\lambda(1-h))}{\bar{\mu}+\lambda\left(1-\beta^{*}(\lambda(1-h))\right)} .
$$


Applying Takacs' lemma [16] to (A.2) we find that if $\rho=\lambda \bar{b}\left(\frac{\bar{\mu}+\lambda}{\bar{\mu}}\right)<1$ :

$$
\begin{aligned}
h(s) & =\sum_{n=1}^{\infty} \frac{\left(\left[\bar{\mu}_{1} s^{-1}+\bar{\mu}_{2} s\right]\left[r_{1} s+r_{2} s^{-1}\right]\right)^{n} \lambda^{n-1}}{n !} \int_{0}^{\infty} e^{-\lambda t} t^{n-1} d S^{n^{*}}(t) \\
& =E\left(\left[\bar{\mu}_{1} s^{-1}+\bar{\mu}_{2} s\right]^{n}\left[r_{1} s+r_{2} s^{-1}\right]^{n}\right) .
\end{aligned}
$$

From (A.1) and (A.3) it follows that

$$
g(s)=E\left(\left[r_{1} s+r_{2} s^{-1}\right]^{n-1}\left[\bar{\mu}_{1} s^{-1}+\bar{\mu}_{2} s\right]^{n}\right) .
$$

The validity of the second statement it can be seen by considering the equation

$$
g(s)=\left[\bar{\mu}_{1} s^{-1}+\bar{\mu}_{2} s\right] \breve{\beta}^{*}\left(g(s) s, g(s) s^{-1}\right) .
$$

Proof of Lemma 6: For $|s|=1$

$$
\begin{aligned}
& S_{1}=\left\{z_{1}: z_{1}=\left[r_{1}+r_{2} s^{2}\right] E\left(\left|r_{1} s+r_{2} s^{-1}\right|^{2 n-2}\right)\right\}, \\
& S_{2}=\left\{z_{2}: z_{2}=\left[r_{1} s^{-2}+r_{2}\right] E\left(\left|r_{1} s+r_{2} s^{-1}\right|^{2 n-2}\right)\right\} .
\end{aligned}
$$

Let,

$$
\begin{aligned}
& U_{1}=\left\{u_{1}: u_{1}=r_{1}+r_{2} s^{2},|s|=1\right\}=\left\{u_{1}: u_{1}=r_{1}+r_{2} e^{2 i \phi}, 0 \leq \phi \leq 2 \pi\right\}, \\
& U_{2}=\left\{u_{2}: u_{2}=r_{1} s^{-2}+r_{2},|s|=1\right\}=\left\{u_{2}: u_{2}=r_{1} e^{-2 i \phi}+r_{2}, 0 \leq \phi \leq 2 \pi\right\} .
\end{aligned}
$$

Clearly, $U_{1}, U_{2}$ are smooth, simple contours, and if $s$ traverses the unit circle once anticlockwise, $U_{1}$ is traversed twice anticlockwise and $U_{2}$ is traversed twice clockwise. Note also that for $|s|=1$,

$$
0<\left|r_{1}-r_{2}\right|=|| r_{1} s|-| r_{2} s^{-1}|| \leq\left|r_{1} s+r_{2} s^{-1}\right| \leq\left|r_{1} s\right|+\left|r_{2} s^{-1}\right|=r_{1}+r_{2}=1 .
$$

Since $n \geq 1$ with probability 1 , it follows that

$$
0<\left(r_{1}-r_{2}\right)^{2} \leq E\left(\left|r_{1} s+r_{2} s^{-1}\right|^{2 n-2}\right) \leq 1 .
$$

Consequently, $S_{1} \subset\left\{z_{1}: \operatorname{Re}\left(z_{1}\right)>0\right\}$. Moreover, since for $s= \pm i$,

$$
\left(r_{1} s^{-2}+r_{2}\right) E\left(\left|r_{1} s+r_{2} s^{-1}\right|^{2 n-2}\right)=\left(r_{2}-r_{1}\right) E\left(\left(r_{1}-r_{2}\right)^{2 n-2}\right)<0,
$$

we realize that $z_{2}=0 \notin S_{2}$. Therefore, $S_{1}, S_{2}$ are both simple, smooth contours. 